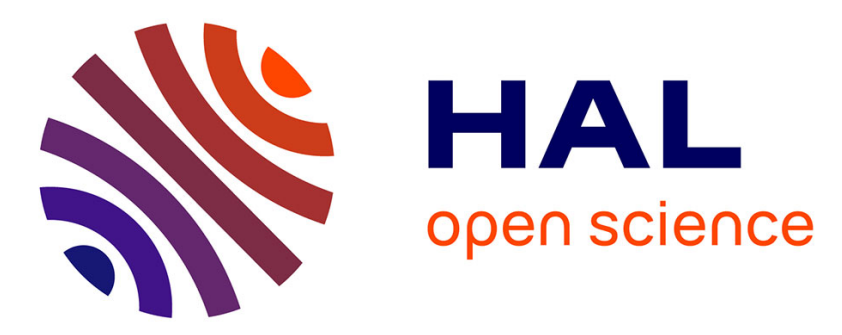

\title{
A tube-based constitutive equation for polydisperse entangled linear polymers
}

Adrien Leygue, Christian Bailly, Roland Keunings

\section{To cite this version:}

Adrien Leygue, Christian Bailly, Roland Keunings. A tube-based constitutive equation for polydisperse entangled linear polymers. Journal of Non-Newtonian Fluid Mechanics, 2006, 136 (1), pp.1-16. 10.1016/j.jnnfm.2006.01.013 . hal-01004957

\section{HAL Id: hal-01004957 https://hal.science/hal-01004957}

Submitted on 29 Apr 2018

HAL is a multi-disciplinary open access archive for the deposit and dissemination of scientific research documents, whether they are published or not. The documents may come from teaching and research institutions in France or abroad, or from public or private research centers.
L'archive ouverte pluridisciplinaire HAL, est destinée au dépôt et à la diffusion de documents scientifiques de niveau recherche, publiés ou non, émanant des établissements d'enseignement et de recherche français ou étrangers, des laboratoires publics ou privés. 


\title{
A tube-based constitutive equation for polydisperse entangled linear polymers
}

\author{
A. Leygue ${ }^{a}$, C. Bailly ${ }^{b, *}$, R. Keunings ${ }^{a}, *$ \\ ${ }^{a}$ CESAME, Université Catholique de Louvain, B-1348 Louvain-la-Neuve, Belgium \\ ${ }^{\mathrm{b}}$ Unité de Chimie et de Physique des Hauts Polymères, Université Catholique de Louvain, B-1348 Louvain-la-Neuve, Belgium
}

\begin{abstract}
We present a tube-based constitutive model for polydisperse entangled linear polymers. The model is constructed as the non-linear extension of a linear model [A. Leygue, C. Bailly, R. Keunings, A differential tube-based model for predicting the linear viscoelastic moduli of polydisperse entangled linear polymers, J. Non Newton. Fluid Mech., in press] capable of predicting quantitatively the linear viscoelasticity of polydisperse linear systems. The constitutive equation accounts for the major linear and non-linear phenomena thought to be important in the description of entangled linear polymers: reptation, contour-length fluctuations, thermal constraint release, convective constraint release and chain stretch effects. In the non-linear regime convective constraint release couples the relaxation of the different masses and provides a non-linear mixing rule for the model. The predictive capabilities of the model are tested on published results for mono- and bi-disperse entangled solutions [C. Pattamaprom, R.G. Larson, Constraint release effects in monodisperse and bidisperse polystyrenes in fast transient shearing flows, Macromolecules 34 (2001) 5229-5237; X. Ye, R.G. Larson, C.J. Pattamaprom, T. Shridar, Extensional properties of monodisperse and bidisperse polystyrene solutions, J. Rheol. 47 (2) (2003) 443-468], both in shear and extension.
\end{abstract}

Keywords: Entangled linear polymers; Constitutive equation; Polymer mixtures

\section{Introduction}

Following the introduction on the reptation picture by de Gennes [4], Doi and Edwards [5] proposed a first tube-based theory to explain the linear rheology of linear entangled polymers. Variations of the tube model have reached a very high level of maturity and are capable of a quantitative description of the linear viscoelastic properties of linear entangled polymers [6-8]. The key of these successes essentially is an accurate description of the reptation dynamics (if present), the fluctuations of the length of the tube and thermal constraint release phenomena, which is a closure to the tube mean field approach. Accounting for coupling between those effects is of critical importance. For linear polymers, the distinction made between reptation and contour-length fluctuations is somehow arbitrary as they represent different modes of the dynamics of a one-dimensional Rouse chain escaping a tube. This distinc-

\footnotetext{
* Corresponding authors. Tel.: +32 1047 8412; fax: +32 10451593 .

E-mail addresses: bailly@poly.ucl.ac.be (C. Bailly), roland.keunings@inma.ucl.ac.be (R. Keunings)
}

tion is not found in stochastic tube theories where the full chain is modelled $[9,10,7]$. Contour-length fluctuations and reptation have been accounted for by using a modified diffusion process along the primitive path. In those theories, one either considers a time dependent [11] or a position dependent $[12,13,1]$ diffusivity along the primitive path. Thermal constraint release is today well understood through dynamic dilution [14] and Rouse motion of the tube. The complex interplay between those phenomena has been thoroughly investigated $[15,16]$. Double reptation $[17,18]$ provides a simple and efficient method to account for constraint release. It essentially provides a reasonable approximation to Rouse tube motion, especially at early times [7]. The essence of Double reptation has actually been recovered within the implementation of constraint release found in a stochastic full chain reptation model developed by Hua and Schieber [9].

The development of theories for the non-linear response of entangled polymers is much more limited and even in the case of monodisperse linear chains, additional phenomena are still proposed as being important in the description of the flow properties of some systems [19]. The Doi-Edwards (D-E) model [5] was a first attempt to build a tube-based constitutive 
equation. Despite the many assumptions found within the D-E model, mostly for mathematical convenience, the predictions of the model are in very good agreement with experimental data for step deformations. For other types of flow the D-E model fails even in the prediction of qualitative features of the non-linear rheology of entangled systems. Among the failures of the D-E model, the most noticeable is the prediction of a shear banding instability in weakly non-linear flows which has never been observed in experiments. This instability is a direct consequence of the non-monotonic curve one can observe when the steady state shear stress is plotted versus the shear rate. Because reptation is the only relaxation mechanism of the D-E model, all tube segments become fully aligned in the shear direction when the shear rate $\dot{\gamma}$ is larger than the inverse reptation time $1 / \tau_{\mathrm{d}}$, which causes the decrease of the shear stress. The excessive shear thinning of the D-E model prevents it from being used in complex flow simulations without considering an additional Newtonian contribution to the viscosity.

In a first attempt to improve the theory, Marrucci and Grizzuti [20] proposed a constitutive model known as the DoiEdwards-Marrucci-Grizzuti (DEMG) model which, unlike the original D-E theory, did not assume the instantaneous retraction of the chain inside the tube. This incorporation of tube stretching effects at a timescale $\tau_{\mathrm{r}}$, much smaller than the timescale $\tau_{\mathrm{d}}$ of reptation, led to improved predictions of the transient response of the model. In particular, it showed overshoots in both shear stress and first normal stress difference upon startup of shear. Although the inclusion of stretch effects might smear out the shear instability of the D-E model for moderately entangled systems, this approach will inevitably fail as one increases the molecular mass $M$ of the entangled species. Indeed, as the time scale of the one-dimensional Rouse relaxation associated with stretch scales like $M^{2}$, an increase in $M$ can render $\tau_{\mathrm{r}}$ arbitrarily small with respect to $\tau_{\mathrm{d}}$ which scales like $M^{3}$. For highly entangled species, the approximation of instantaneous chain retraction is therefore valid and cannot be held responsible for the shear banding instability of the D-E model.

Finally, in 1996, Marrucci [21] successfully pointed out constraint release as the key to overcome the problem. Although constraint release may be considered to play only a mild role in describing the linear viscoelastic behaviour of a monodisperse material, it has a much greater influence in the non-linear regime where constraint release events may not only be triggered by reptation but also by chain retraction. For flows where the deformation rate $\dot{\gamma}$ lies between $1 / \tau_{\mathrm{d}}$ and $1 / \tau_{\mathrm{r}}$ the rate of constraint release would then be proportional to $\dot{\gamma}$, which is the rate of retraction necessary to maintain the chain at its equilibrium length. This type of constraint release is called convective constraint release (CCR) as, from the point of view of the chain, constraints are convected by the flow along the tube. The first attempts by Ianniruberto and co-workers [22] and Marrucci et al. [23] to incorporate CCR into a constitutive equation were focusing on flow regimes where $\dot{\gamma} \leq 1 / \tau_{\text {r }}$ and assumed complete chain retraction. As the rate of CCR has then to be computed from the relative alignment of the microstruc- ture with the flow field, it is difficult to obtain a general CCR formulation that does not rely on a switch function to turn CCR off when it would yield unrealistic predictions. The thermodynamical validity of such formulations of CCR for differential constitutive equations has been discussed by Leygue et al. [24].

More successful constitutive equations account explicitly for chain stretch and compute the rate of CCR directly from the rate of chain retraction. Using a very detailed integral model, Mead et al. [25] showed how CCR, combined with tube length fluctuations and stretch effects, could prevent the excessive shear thinning and solve other problems of the D-E model. Their approach however relied on a separate representation of the average stretch and orientation of the tube and still needed a switch function to balance CCR between the relaxation of stretch and orientation.

Recently, Marrucci and Ianniruberto [26] proposed a promising single segment model that incorporates both chain stretch and CCR in a coupled representation. This new model is simple enough to be easily used for complex flow simulations [27] but yet retains the necessary physics.

The construction of microstructural constitutive models for entangled systems is a process in which many mathematical approximations have to be made in order to obtain a closed set of partial differential equations. From this angle, stochastic models offer an attractive approach, where the mathematical complexity of the approximations is partially replaced by the numerical complexity and cost of stochastic differential equations. Based on microstructural mechanical models, successful full chain stochastic reptation models have been proposed by Hua et al. [9] and Masubuchi et al. [10]. These models are very useful to understand the influence of some physical effects but their numerical complexity still prevents them from being used in complex flow simulations.

In the present text, we present the CRAFT model; a new tube-based constitutive equation for entangled linear polymers. We first recall a simple linear theory for the prediction of the linear viscoelastic properties of polydisperse systems [1]. Then, we extend this theory to a full constitutive equation for entangled linear polymers. This constitutive model incorporates, in a full chain approach, the major molecular mechanisms thought to be important to describe the flow of entangled polymers: reptation, contour-length fluctuations, thermal and convective constraint release, chain stretch and finite extensibility of the polymeric chains. Thermal and convective constraint release are modelled in a unified fashion where the latter appears as a non-linear addition to the former. Additionally, the linearization of our model is equivalent to the original linear theory.

A central feature is that most of the parameters of the constitutive model can be identified and understood from the linear viscoelastic response. For polydisperse systems, constraint release actually provides the mixing rule in both the linear and non-linear regimes, with no additional parameter. The acronym CRAFT stands for constraint release on average full tensorial chain, the main feature of our model is its implementation of constraint release, on a tensorial representation of the averaged prim- 
itive chains. Finally, we analyse the predictions of the CRAFT constitutive equation and compare them with recently published experimental data for concentrated polystyrene solutions in various rheometrical flows. We show that the CRAFT model is able, using a single set of material parameters, to quantitatively predict both linear and non-linear rheological properties of linear entangled polymers.

\section{A linear model for linear entangled polymers}

Most of today's quantitative theories $[8,28,29,6,7,30]$ that predict the relaxation modulus as a function of the molecular weight distribution and a few material parameters are mathematically non-linear. This feature does not allow for their direct extension to a full constitutive equation for predicting the nonlinear rheology. Starting from a mathematically linear model for predicting the linear viscoelasticity of linear polymers [1], we build the CRAFT constitutive equation as a non-linear extension. As non-linear relaxation phenomena are incorporated into the constitutive equation we always require that they do not change its linear response. The model is therefore able to describe both the linear and non-linear rheology of entangled systems. In this section, we briefly recall the simple linear model in the polydisperse case.

In a polydisperse mixture of entangled linear polymers, let us consider the chains of a given mass $M^{(i)}$ among the $N$ masses. Let $s$ be a curvilinear coordinate along the primitive path. For simplicity, all lengths are made non-dimensional with respect to half the equilibrium length of the primitive path. Therefore we have $-1 \leq s \leq 1$. We then define $P_{\gamma}^{f(i)}(t, s)$ as the probability for a chain segment of coordinate $s$ of being in a tube segment that is older than $t$. This tube survival probability decreases in time under the combined effects of reptation, contour-length fluctuations and thermal constraint release. Although the spectrum of relaxation times due to reptation and contour-length fluctuations is relatively narrow for a given mass, a polydisperse environment will yield a broad spectrum of relaxation times for $P_{\gamma}^{f(i)}(t, s)$. The tube survival probability is therefore approximated by a sum of modes corresponding to the dominant relaxation times induced by thermal constraint release:

$P_{\gamma}^{f(i)}(t, s) \approx \sum_{j=1}^{N_{\mathrm{CR}}} w_{j} P_{\gamma}^{f(i, j)}(t, s)$,

where $N_{\mathrm{CR}}$ is representative of the number of characteristic relaxation times $\tau_{j}$ induced by thermal constraint release and $w_{j}$ represent the relative weights of those times. This description in terms of modes of constraint release implies that, for a fixed (i), the contribution of reptation and contour-length fluctuations to the dynamics of $P_{\gamma}^{f(i, j)}(t, s)$ is identical for all $(j)$.

Let us now focus on the dynamics of $P_{\gamma}^{f(i, j)}(t, s)$. Following [13], reptation and fluctuations are modelled together by a diffusion operator with a variable diffusivity along the coordinate $s$. Additionally, thermal constraint release is introduced through a linear relaxation term with a characteristic time $\tau_{j}$. The resulting differential problem for $P_{\gamma}^{f(i, j)}(t, s)$ reads:

$\frac{\partial P_{\gamma}^{f(i, j)}}{\partial t}=\frac{\partial}{\partial s}\left(\alpha_{\mathrm{d}}^{f(i)}(s) \frac{\partial}{\partial s} P_{\gamma}^{f(i, j)}\right)-\frac{1}{\tau_{j}} P_{\gamma}^{f(i, j)}$,

$P_{\gamma}^{f(i, j)}(t,-1)=0$,

$P_{\gamma}^{f(i, j)}(t, 1)=0$ for $t>0$,

$P_{\gamma}^{f(i, j)}(0, s)=1$ for $-1<s<1$.

Contour-length fluctuations are assumed to modify the diffusion coefficient $\alpha_{\mathrm{d}}^{f(i)}(s)$ up to a depth of order $\sqrt{M^{(i)} / M_{\mathrm{e}}}$, where $M_{\mathrm{e}}$ is the entanglement molecular weight. The expression for $\alpha_{\mathrm{d}}^{f(i)}(s)$ reads:

$\alpha_{\mathrm{d}}^{f(i)}(s)=\frac{4}{K_{\mathrm{d}} \pi^{2} M^{(i)^{3}}} \frac{K_{\mathrm{f}}^{2} M_{\mathrm{e}}}{M^{(i)}(1-s)^{2}}$,

if $s>\left(1-K_{\mathrm{f}} \sqrt{\frac{M_{\mathrm{e}}}{M^{(i)}}}\right)$,

$\frac{4}{K_{\mathrm{d}} \pi^{2} M^{(i)^{3}}} \frac{K_{\mathrm{f}}^{2} M_{\mathrm{e}}}{M^{(i)}(1-s)^{2}}, \quad$ if $s<\left(K_{\mathrm{f}} \sqrt{\frac{M_{\mathrm{e}}}{M^{(i)}}}-1\right)$

$\frac{4}{K_{\mathrm{d}} \pi^{2} M^{(i)^{3}}}$ otherwise,

where $K_{\mathrm{d}}$ is a material parameter. The adjustable parameter $K_{\mathrm{f}}$ is close to unity and controls the depth of the contour-length fluctuations within the model.

The relaxation times $\tau_{j}$, induced by thermal constraint release, and their associated weights $w_{j}$ are such that they yield a constraint release kernel as close as possible to the constraint release kernel that double reptation would induce:

$\sum_{j=0}^{N_{\mathrm{CR}}} w_{j} \exp \left(\frac{-t}{\tau_{j}}\right) \approx\left(\sum_{i=1}^{N} \frac{\phi^{(i)}}{2} \int_{-1}^{1} P_{0}^{f(i)}(t, s) \mathrm{d} s\right)^{\gamma}$.

The parameter $\gamma$ is a mixing exponent of order unity and the functions $P_{0}^{f(i)}(t, s)$ are the tube survival probabilities with thermal constraint release neglected. They are computed from the following differential problem:

$\frac{\partial}{\partial t} P_{0}^{f(i)}=\frac{\partial}{\partial s}\left(\alpha_{\mathrm{d}}^{f(i)}(s) \frac{\partial}{\partial s} P_{0}^{f(i)}\right)$,

$P_{0}^{f(i)}(t,-1)=0$,

$P_{0}^{f(i)}(t, 1)=0$ for $t>0$,

$P_{0}^{f(i)}(0, s)=1$ for $-1<s<1$.

The relaxation modulus is proportional to the average tube survival probability over all masses:

$G(t)=G_{N}^{0} \sum_{i=1}^{N} \sum_{j=1}^{N_{\mathrm{CR}}} \phi^{(i)} w_{j} \frac{1}{2} \int_{-1}^{1} P_{\gamma}^{f(i, j)}(t, s) \mathrm{d} s$,

where $G_{N}^{0}$ is the plateau modulus, and $\phi^{(i)}$ is the volume fraction of mass $(i)$. In order to make quantitative predictions of the 
linear viscoelastic moduli at high frequencies, one should also account for the Rouse relaxation modes of the chains. The Rouse relaxation time $\tau_{\mathrm{r}}$ of an unentangled chain is given by:

$\tau_{\mathrm{r}}=K_{\mathrm{r}} M^{2}$,

where $K_{\mathrm{r}}$ is a linear material parameter. Following van Ruymbeke et al. [6], the Rouse spectrum $G_{\mathrm{r}}(t)$ for that entangled chain writes:

$$
\begin{aligned}
G_{\mathrm{r}}(t)= & G_{N}^{0}\left(\sum_{p=Z+1}^{\infty} \frac{1}{Z} \exp \left(-\frac{p^{2}}{\tau_{\mathrm{r}}}\right)\right. \\
& \left.+\frac{1}{3} \sum_{p=1}^{Z} \frac{1}{Z} \exp \left(-\frac{p^{2}}{\tau_{\mathrm{r}}}\right)\right) .
\end{aligned}
$$

The integer $Z$ is defined as the closest integer to the ratio $M / M_{\mathrm{e}}$. The Rouse modulus of each mass $M^{(i)}$ is to be superposed to the reptation relaxation modulus using a linear mixing rule.

Although $K_{\mathrm{d}}, K_{\mathrm{r}}, M$ and $M_{\mathrm{e}}$ are linked through the underlying segmental dynamics and should not be specified independently, they are considered as independent parameters in this work.

The set of equations presented in this section is closed and forms a mathematically linear model that can predict quantitatively the linear viscoelastic response of entangled linear polymers. In the following sections, we will extend this model to a full constitutive equations for non-linear flow regimes.

\section{Construction of the CRAFT constitutive equation}

For the sake of simplicity we will present the construction of the CRAFT model for the monodisperse case only and for only one characteristic time of thermal constraint release. This simplification allows us to discard the $(i, j)$ superscripts we had to consider in the previous section. The polydisperse case with a full thermal constraint release spectrum will be presented as a natural extension in a latter section.

The variables of the CRAFT model are the components of a second order conformation tensor $\mathbf{c}(t, s)$ defined along the coordinate $s$ of the primitive path. The dynamics of this tensor is controlled by a PDE which accounts for the following phenomena:

- affine deformation of the micro-structure;

- reptation of the primitive chain;

- contour-length fluctuations;

- relaxation of tube stretch;

- thermal and convective constraint release;

- finite extensibility of the polymeric chain.

In the next sections, we will first define some additional notations and then consider the contribution of each phenomenon to the evolution of $\mathbf{c}(t, s)$.

\subsection{Variables and notations}

Let us consider a single polymeric chain, trapped in a fixed tube, as shown in Fig. 1. Both the primitive chain and the tube exist in real space (3D), but can be referenced through a single parametric coordinate $s$. For simplicity, all lengths in real space are made non-dimensional with respect to half of the equilibrium length of the primitive path. At equilibrium, the non-dimensional length of the primitive path is therefore 2 .

Let $x(t, s)$ be the position of the primitive chain along the tube, where $s(-1 \leq s \leq 1)$ is a Lagrangian coordinate along the chain. At equilibrium, $s$ is a curvilinear coordinate which implies that $(x-s)$ is a constant. The position of the origin $x=0$ is actually arbitrary. The position of the tube in real space is defined by the vector $\mathbf{r}(x)$, and by definition of $x$ and $\mathbf{r}$ we have:

$\left\|\frac{\partial \mathbf{r}}{\partial x}\right\|=1$.

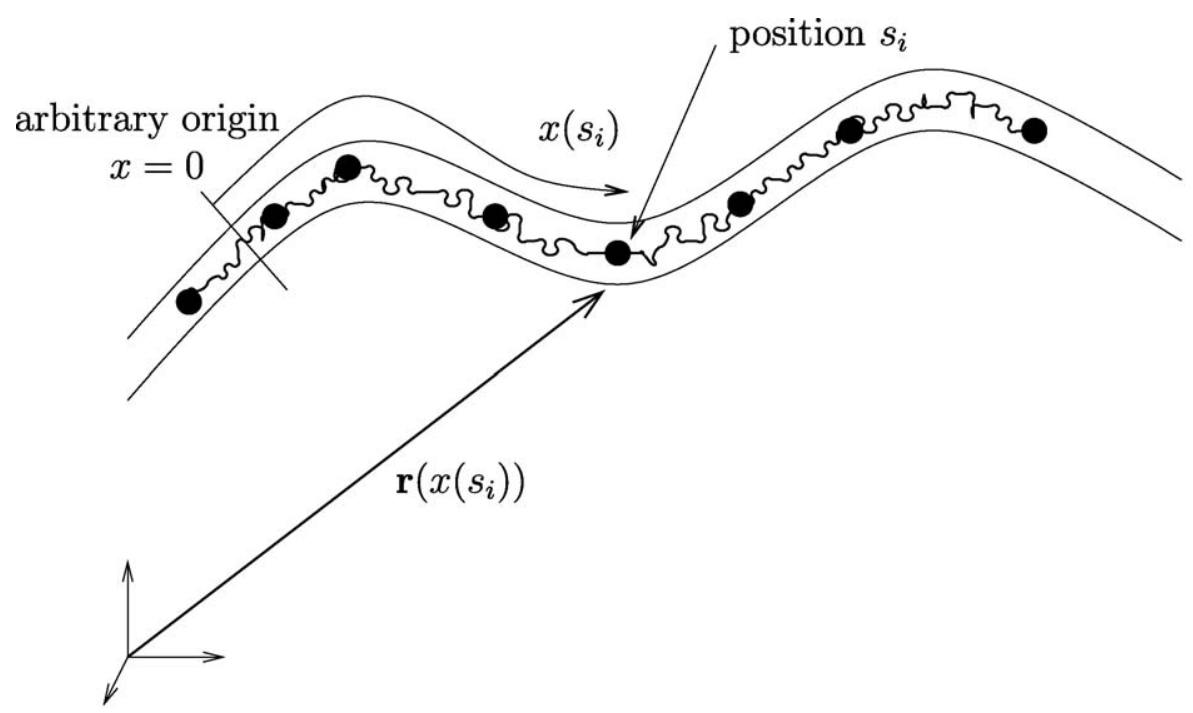

Fig. 1. Illustration of the notations used to build the CRAFT model. 
For a system composed of many chains, let us define the secondorder conformation tensor $\mathbf{c}(t, s)$ as:

$\mathbf{c}(t, s)=3\left\langle\frac{\partial \mathbf{r}}{\partial s} \frac{\partial \mathbf{r}}{\partial s}\right\rangle$,

where $\langle\cdot\rangle$ is the statistical ensemble average over all chains. This definition of $\mathbf{c}$ yields:

$\operatorname{tr} \mathbf{c}=3\left\langle\left(\frac{\partial x}{\partial s}\right)^{2}\right\rangle$,

which is the average local stretch along the primitive path. We now have to propose an evolution equation for the set of tensors $\mathbf{c}(t, s)$ as well as a way to compute the stress tensor.

\subsection{Affine deformation}

We decide to couple the dynamics $\mathbf{c}(t, s)$ to the velocity field with the assumption of affine deformation of the micro-structure. The induced strain measure is therefore the classical affine strain measure. At the microstructural level, this yields the following evolution equation for the tangent vector $\frac{\partial \mathbf{r}}{\partial s}$ :

$\frac{D}{D t} \frac{\partial \mathbf{r}}{\partial s}=\boldsymbol{\kappa} \cdot \frac{\partial \mathbf{r}}{\partial s}$,

where $\boldsymbol{\kappa}$ is the velocity gradient. Through direct substitution of Eq. (14) in Eq. (12), we find the following evolution equation for $\mathbf{c}(t, s)$ :

$\frac{\partial \mathbf{c}}{\partial t}(t, s)=\boldsymbol{\kappa} \cdot \mathbf{c}+\mathbf{c} \cdot \boldsymbol{\kappa}^{T}$,

or

$\stackrel{\nabla}{\mathbf{c}}(t, s)=0$

When all relaxation phenomena can be neglected (e.g. in a step deformation) the evolution of $\mathbf{c}(t, s)$ is governed by the upper convected time derivative and $\mathbf{c}(t, s)$ is therefore equal to the Finger strain tensor.

\subsection{Reptation and fluctuations dynamics}

To incorporate reptation within the model, we postulate that the dissipative dynamics of reptation is governed by the same operator that governs the relaxation of $P_{\gamma}^{f}(t, s)$ :

$\frac{\partial}{\partial s}\left(\alpha_{\mathrm{d}}(s) \frac{\partial \cdot}{\partial s}\right)$.

Through this differential operator, we explicitly take into account the connectivity of the chain and the physical process of reptation. Hence, we build a full chain model rather than a single segment model. As reptation is a diffusion process in real space which we solve in parametric space $s$, the onset of chain stretch should be accounted for in the change of variable from $x$ to $s$. To ensure a constant rate of diffusion in real space, Graham et al. [13] modify the diffusion operator as the stretch increases. We do agree with the physics and the mathematics behind this proposal, but we choose not to incorporate it in our model. The reason is two-fold. In the first place, Marrucci has showed [21] that convective constraint release (CCR) becomes the dominant relaxation mechanism as soon as the rate of deformation is greater than the inverse reptation time. As the stretch relaxation time is smaller than the reptation time, this occurs even before the onset on chain stretch. The renormalisation of the diffusion operator due to chain stretch will therefore be significant for flow regimes where reptation has already been superseded by CCR. Second, we believe that even before the onset of chain stretch, anisotropy effects within the entangled network are likely to appear and modify the diffusion process in a more significant way.

At the chain ends, we assume that the primitive chain is always fully relaxed in both stretch and orientation. For $s= \pm 1$, the vectors $\frac{\partial \mathbf{r}}{\partial s}$ are therefore uniformly distributed on the unit sphere, which yields:

$\mathbf{c}(t, \pm 1)=\delta$.

The validity of this assumption can be questioned as it implies that no matter the strength of the flow, the chain ends will always be fully relaxed. Ignoring anisotropy effects, we can nevertheless assume that this assumption is valid for flow rates up to the inverse of the segmental time $\tau_{e}$.

\subsection{Chain stretch dynamics}

In this section, we address the problem of describing the relaxation of chain stretch and its coupling with orientation.

Let us model the retraction of the chain as due to a onedimensional Rouse motion of the chain inside the tube. The projection along the primitive path of the microscopic force balance at position $s$ yields the following evolution equation for $x(t, s)$ :

$\frac{\partial x}{\partial t}=\alpha_{\mathrm{r}} \frac{\partial^{2} x}{\partial s^{2}}$

$\frac{\partial}{\partial s} x(t, \pm 1)=1$

where $s$ is the Lagrangian coordinate along the chain, $x(t, s)$ is the curvilinear position for the chain along the tube and $\alpha_{\mathrm{r}}$ is a characteristic diffusion constant, scaling like the inverse of the square of the molecular mass. Eq. (20) is a boundary condition expressing that chain ends are always fully relaxed in stretch. The steady state solution of (19) is linear in $s$ and the equilibrium length of the chain is 2 .

Under the assumption that the tube is a fixed object, i.e. reptation phenomena are slow compared to the relaxation of stretch along the primitive path, we would like to find the contribution of the chain dynamics (19) to the evolution of c. Considering the definition of $\mathbf{c}$, let us first try to obtain a tractable expression for the following quantity:

$\frac{\partial}{\partial t}\left(\frac{\partial \mathbf{r}(x(t, s))}{\partial s} \frac{\partial \mathbf{r}(x(t, s))}{\partial s}\right)$. 
From (19), applying chain differentiation we can approximate (21) as:

$$
\begin{aligned}
\frac{\partial}{\partial t}\left(\frac{\partial \mathbf{r}}{\partial s} \frac{\partial \mathbf{r}}{\partial s}\right) \approx & \frac{\alpha_{\mathrm{r}}}{2} \frac{\partial}{\partial s}\left(\frac{\partial x}{\partial s}\right)^{2} \frac{\partial}{\partial s}\left(\frac{\partial \mathbf{r}}{\partial x} \frac{\partial \mathbf{r}}{\partial x}\right) \\
& +\alpha_{\mathrm{r}}\left(\frac{\partial \mathbf{r}}{\partial x} \frac{\partial \mathbf{r}}{\partial x}\right) \frac{\partial^{2}}{\partial s^{2}}\left(\frac{\partial x}{\partial s}\right)^{2}
\end{aligned}
$$

The only approximation made to obtain the previous expression is:

$$
\frac{\partial^{2}}{\partial s^{2}}\left(\frac{\partial x}{\partial s}\right)^{2}=2 \frac{\partial x}{\partial s} \frac{\partial^{2}}{\partial s^{2}} \frac{\partial x}{\partial s}+2\left(\frac{\partial^{2} x}{\partial s^{2}}\right)^{2} \approx 2 \frac{\partial x}{\partial s} \frac{\partial^{2}}{\partial s^{2}} \frac{\partial x}{\partial s},
$$

The purpose of this approximation is to obtain a closed form for the evolution equation of $\left(\frac{\partial \mathbf{r}}{\partial s} \frac{\partial \mathbf{r}}{\partial s}\right)$.

The term which is neglected is the square of a curvature term and the approximation can therefore be interpreted as a mild curvature assumption. The validity of this approximation has been successfully tested for a single chain relaxing in a tube after a step strain in shear or extension [31].

To go from the relaxation of a single chain to the relaxation of $\mathbf{c}$, we need to average (22) over the ensemble of chains. In this process, we approximate the average of products/ratios by the products/ratios of the averaged quantities. These closure approximations are required in order to obtain a closed set of equations for the stretch dynamics. The resulting evolution equation for $\mathbf{c}$ is the following:

$$
\frac{\partial \mathbf{c}}{\partial t}=\frac{\alpha_{\mathrm{r}}}{2} \frac{\partial \operatorname{tr} \mathbf{c}}{\partial s} \frac{\partial}{\partial s} \frac{\mathbf{c}}{\operatorname{tr} \mathbf{c}}+\alpha_{\mathrm{r}} \frac{\mathbf{c}}{\operatorname{tr} \mathbf{c}} \frac{\partial^{2} \operatorname{tr} \mathbf{c}}{\partial s^{2}} .
$$

As taking the trace of the previous expression makes the first term of the right-hand side disappear, we see that the second term alone governs the relaxation of stretch along the chain. More specifically, stretch relaxes through a diffusion process with a time scale of order $\frac{1}{\alpha_{\mathrm{r}}}$. This term also couples the relaxation of the diagonal and non-diagonal components of $\mathbf{c}$ in such a way that it does not modify the purely orientational part: $\frac{\mathbf{c}}{\operatorname{tr} \mathbf{c}}$. Indeed, if $\frac{\partial \mathbf{c}}{\partial t}=\alpha_{\mathrm{r}} \frac{\mathbf{c}}{\operatorname{tr} \mathbf{c}} \frac{\partial^{2} \operatorname{tr} \mathbf{c}}{\partial s^{2}}$ then $\frac{\partial}{\partial t}\left(\frac{\mathbf{c}}{\operatorname{tr} \mathbf{c}}\right)=0$.

The first term on the right-hand side of (23) influences the local orientation only. It can be interpreted as a transport term along the $s$ coordinate, wherein the velocity is proportional to the stretch gradient and the transported quantity is purely orientational: $\frac{\mathbf{c}}{\operatorname{tr} \mathbf{c}}$. As chain retraction occurs, it slows down the overall relaxation as the orientation of the chain is transfered from the inner segments to the outer, and more relaxed, segments.

In view of the many approximations needed to obtain Eq. (23), one might rely on the bracket formalism of non-equilibrium thermodynamics [32] to obtain an alternative phenomenological expression for the evolution of $\mathbf{c}$ induced by the chain stretch dynamics. The form of Eq. (23) provides however a guide to build the appropriate dissipation bracket. A simple expression for the dissipation bracket [33] yields the following expression:

$$
\frac{\partial \mathbf{c}}{\partial t}=\alpha_{\mathrm{r}} \mathbf{c} \frac{\partial}{\partial s} \frac{1}{\operatorname{tr} \mathbf{c}} \frac{\partial \operatorname{tr} \mathbf{c}}{\partial s}+\alpha_{\mathrm{r}} \frac{\mathbf{c}}{\operatorname{tr} \mathbf{c}} \frac{\partial^{2} \operatorname{tr} \mathbf{c}}{\partial s^{2}} .
$$

This expression is very close to Eq. (23) and is compatible with the bracket formalism of non-equilibrium thermodynamics. Future work should focus on its evaluation and its comparison with Eq. (23) in different flow regimes.

\subsection{Constraint release}

Generalising our approach of thermal constraint release from section 2, we assume it is possible to model both thermal and convective constraint release phenomena as a local relaxation process where the rate of relaxation due to CCR is computed from the dynamics of stretch relaxation. Furthermore, we assume that thermal and convective constraint release are independent allowing us to sum their rates.

The procedure to compute the rate of thermal constraint release has been detailed in Section 2. For the sake of clarity, we will assume here that thermal constraint release can be described with a single relaxation time $\tau_{\mathrm{cr}}$. The remaining issue is therefore to give a valid expression of the rate of constraint release $f_{\text {ccr }}$ under the following constraints:

- As the constitutive equation must be applicable in any type of flow and in any coordinate system, only the invariants of c can be used for expressing $f_{\text {ccr }}$.

- The velocity gradient cannot be used explicitly for computing $f_{\text {ccr }}$ without special attention. From a thermodynamical point of view, this would bring an additional coupling between the velocity and the variables describing the microstructure, which has to be accounted for in the stress tensor [32]. Furthermore, such a coupling might lead to a negative rate of entropy production in some flows and a switch function is therefore needed to prevent this [24].

- In the linear regime, the expression for $f_{\text {ccr }}$ must vanish.

- In the non-linear regime, $f_{\text {ccr }}$ should account for CCR. Its value has then to governed by stretch relaxation.

A first possibility to compute the rate of CCR would be to follow Mead et al. [25] and simply take the rate of convection of mesh of entanglements, relative to the rate at which the chain is stretched. Neglecting some pre-factors of order unity, this would yield the following expression:

$f_{\text {crr }}(t)=-\frac{\int_{-1}^{1} \alpha_{\mathrm{r}}\left(\frac{\partial^{2}}{\partial s^{2}}\right) \operatorname{tr} \mathbf{c}(t, s) \mathrm{d} s}{\int_{-1}^{1} \operatorname{tr} \mathbf{c}(t, s) \mathrm{d} s}$.

Although this expression is quite appealing, it can lead to negative rates of CCR in some reversing flows such as large amplitude oscillatory shear (LAOS) flows. For well entangled systems, the reptation time $\tau_{\mathrm{d}}$ and stretch relaxation time $\tau_{\mathrm{r}}$ can be well separated. If the characteristic time of the LAOS flow $v^{-1}$ is such that $\tau_{\mathrm{r}} \ll v^{-1} \ll \tau_{\mathrm{d}}$, the microstructure can be fully oriented but not stretched at all when the maximum strain is reached. When the 
flow starts to reverse, the flow kinematics will tend to compress the chains below their equilibrium length. The stretch relaxation processes will therefore tend to increase the length of the chains to preserve their length, leading to a negative value for $f_{\mathrm{ccr}}(t)$. Therefore we propose the following more general expression for the rate of CCR:

$f_{\text {ccr }}(t)=-\frac{\left.\int_{-1}^{1}\left(\frac{D}{D t}\right) a(\mathbf{c}(t, s))\right|_{\text {stretch }} \mathrm{d} s}{\int_{-1}^{1} a(\mathbf{c}(t, s)) \mathrm{d} s}$,

where $a(\mathbf{c}(s))$ is the contribution from the chain segments with position $s$ to the local free energy density. The notation $\left.\frac{D}{D t}\right|_{\text {stretch }}$ represents the Lagrangian variation of a quantity due to the relaxation of stretch. Under the hypothesis that sub-chains are Gaussian, $a(\mathbf{c})$ takes the form [32]:

$a(\mathbf{c})=G_{N}^{0}(\operatorname{tr}(\mathbf{c})-\ln \operatorname{det}(\mathbf{c}))$,

which is the free energy one would use if c was describing Hookean dumbbells. Finite extensibility issues have also been neglected in the definition of $a(\mathbf{c})$. In the next section, when finite extensibility will be taken into account, we will use the free energy corresponding to FENE-P dumbbells. In Appendix A, we show that Eq. (26) always yields a positive rate of constraint release. Except for reversing flows where the flow might tend to compress the polymeric coils, the first term of Eq. (27) is always dominant and the rate of CCR (26) actually reduces to Eq. (25).

Finally we want to account for chain stretch effects, which locally reduce the rate of relaxation through CCR. Indeed, if one assumes that the tube persistence length is fixed, a stretched portion of the chain will have more constraint release sites than under equilibrium condition. Consequently, we define the local rate of relaxation due to constraint release $f_{\mathrm{cr}}^{\text {loc }}$ as the sum of the rates of thermal and convective constraint release divided by the local stretch ratio:

$f_{\mathrm{cr}}^{\mathrm{loc}}(t, s)=\left(\frac{1}{\tau_{\mathrm{cr}}}+f_{\mathrm{ccr}}(t)\right) \frac{3}{\operatorname{tr} \mathbf{c}(t, s)}$.

A more detailed discussion of this modification of the rate of CCR can be found in [13].

\subsection{The CRAFT model for monodisperse systems}

In this section, we propose a model containing all the elements we presented so far plus finite extensibility effects. Additionally, we provide an expression for the stress tensor $\boldsymbol{\tau}_{p}$. Based on the assumption that one can simply add all the terms we proposed for the time evolution of $\mathbf{c}$, we propose the following constitutive equation:

$$
\begin{aligned}
\underset{\mathbf{c}}{\nabla}(t, s)= & \frac{\partial}{\partial s}\left(\alpha_{\mathrm{d}}^{f}(s) \frac{\partial}{\partial s}(f \mathbf{c})\right)+\alpha_{\mathrm{r}} \frac{\mathbf{c}}{\operatorname{tr} \mathbf{c}} \frac{\partial^{2}}{\partial s^{2}}(f \operatorname{tr} \mathbf{c}) \\
& +\frac{\alpha_{\mathrm{r}}}{2} \frac{\partial(f \operatorname{tr} \mathbf{c})}{\partial s} \frac{\partial}{\partial s}\left(\frac{\mathbf{c}}{\operatorname{tr} \mathbf{c}}\right)-\left(\frac{1}{\tau_{\mathrm{cr}}}+f_{\mathrm{ccr}}\right) \frac{3}{\operatorname{tr} \mathbf{c}}(f \mathbf{c}-\delta),
\end{aligned}
$$

with,

$\mathbf{c}(t, \pm 1)=\delta$

$f_{\text {ccr }}=-\frac{\left.\int_{-1}^{1}\left(\frac{D a(\mathbf{c})}{D t}\right)\right|_{\text {stretch }} \mathrm{d} s}{\int_{-1}^{1} a(\mathbf{c}) \mathrm{d} s}$,

$f(\mathbf{c})=\frac{b-3}{b-\operatorname{tr} \mathbf{c}}$,

$a(\mathbf{c})=\left(-(b-3) \ln \left(1-\frac{\operatorname{tr} \mathbf{c}}{b}\right)-\ln \operatorname{det}(\mathbf{c})\right)$,

$\boldsymbol{\tau}_{p}=G_{N}^{0} \frac{1}{2} \int_{-1}^{1}(f \mathbf{c}-\boldsymbol{\delta}) \mathrm{d} s$.

In the previous set of equations, $\alpha_{\mathrm{d}}^{f}(s)$ is defined from Eq. (3), while $\alpha_{\mathrm{r}}$ is defined as:

$\alpha_{\mathrm{r}}=\frac{4}{\pi^{2} K_{\mathrm{r}} M^{2}}$,

where $M$ is the molar mass of the polymeric chains. For both the linear and non-linear rheology the model has seven parameters: $K_{\mathrm{d}}, K_{\mathrm{r}}, K_{\mathrm{f}}, M_{\mathrm{e}}, G_{N}^{0}, b$ and $\gamma$. Out of those seven, only $b$ is actually a truly non-linear parameter.

The parameter $b$ is the finite extensibility parameter, such that $\operatorname{tr} \mathbf{c}<b$. Defining $L_{\mathrm{eq}}$ as the equilibrium length of the primitive chain and $L_{\max }$ its maximum length, we have:

$b=3 \frac{L_{\max }^{2}}{L_{\mathrm{eq}}^{2}}=3 N_{k}$,

where $N_{k}$ is the number of Kuhn steps between entanglements at equilibrium.

The parameter $K_{\mathrm{r}}$ is the scaling parameter for the Rouse time $\tau_{\mathrm{r}}$, which controls both the high frequency regime of the linear viscoelastic moduli and the stretch relaxation dynamics. Consequently, this parameter can be identified either on the linear or on the non-linear response of the system. In this work we consider $K_{\mathrm{r}}$ as independent from $K_{\mathrm{d}}$ and $M_{\mathrm{e}}$, and we will preferably identify its value on some non-linear experiment.

\section{Computing numerical predictions}

The CRAFT model has the form of a set of non-linear coupled PDEs along the $s$ coordinate. The equations are coupled through the trace of $\mathbf{c}$ which appears in the stretch relaxation and constraint release terms, but also through the determinant of $\mathbf{c}$ which is found in the expression for $f_{\mathrm{ccr}}$.

Before trying to solve these equations numerically, one can use the symmetry of the problem to reduce the $s$ domain to the interval ]0, 1[. Zero flux boundary conditions are then imposed at $s=0$. To solve the resulting PDEs on the reduced domain, we chose to discretize them along the $s$ coordinate using a finite difference scheme. The discretization has to be fine enough to capture the boundary layer that appears at the chain ends for high deformation rates. Integration in time of the discretized system is then performed using an adaptive Runge-Kutta or Gear ODE solver. This last step should be performed with care, 
as the simulation of high molecular masses or very polydisperse systems is very likely to induce many different time-scales in the ODE, that may differ by orders of magnitude. The use of an ODE solver for stiff problems is therefore highly recommended, especially at high deformation rates.

In its discretized form, the model can be interpreted as a coupled multi-segment constitutive equation where the coupling between the modes is naturally imposed through the discretized differential operators along the primitive path. In this form, the model can be used in a straightforward manner for complex flow calculations. Simulations of coupled multi-segments differential models in complex flows are indeed possible using standard numerical methods for non-Newtonian flows (e.g. Wapperom et al. [34]). In complex flows, the single chain coordinate $s$ of the CRAFT model might be an advantage over the double chain coordinate $s$ and $s^{\prime}$ of the model proposed by Graham et al. [13], as the numerical cost of integrating the equations of the model in many points of the flow domain might become significant.

\section{Predictions in simple shear flow}

In this section, we present the steady state and transient responses of the CRAFT model for simple shear flows. For all the figures, we set the value of $\gamma$ to 1.15 and we define $M_{\mathrm{e}}$ as $M_{\mathrm{e}}=\frac{3 M \tau_{\mathrm{r}}}{\tau_{\mathrm{d}}}$.

In Fig. 2, we report the steady shear stress of the CRAFT model as a function of the Deborah number De $=\dot{\gamma} \tau_{\text {eff }}$, where $\dot{\gamma}$ is the shear rate and $\tau_{\text {eff }}=\frac{\eta_{0}}{G_{N}^{0}}$. Finite extensibility has been neglected as $b \rightarrow \infty$. We see that when the ratio $\tau_{\mathrm{d}} / \tau_{\mathrm{r}}$ is small enough, the curve is monotonic, but as this ratio gets bigger a shallow maximum appears in the curve. This feature is however smeared out as soon as polydispersity comes into play. We believe that a phenomenological modification of the model in order to tune the effectiveness of convective constraint release, such as the introduction the $\beta$ parameter found in [26], could

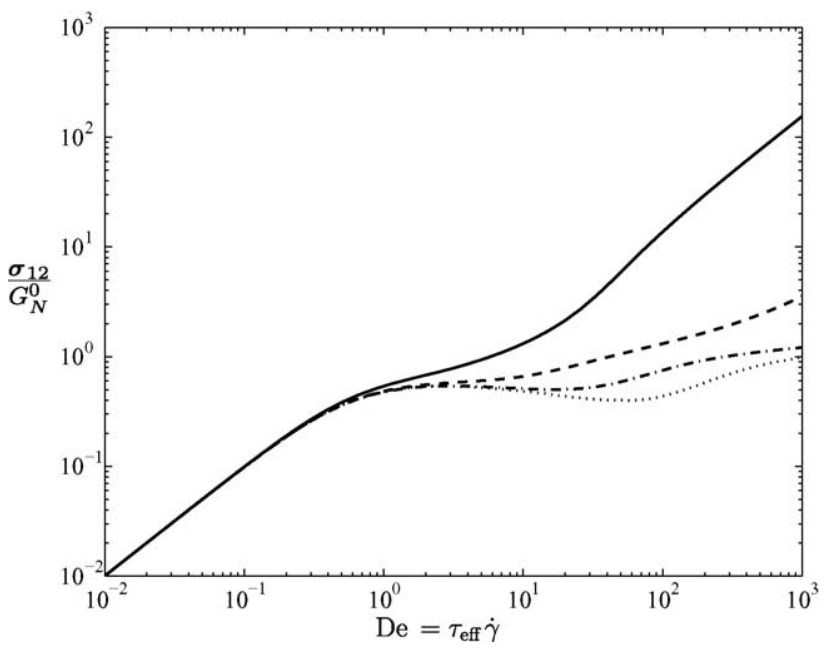

Fig. 2. Steady state shear stress as a function of the Deborah number. The four curves correspond to different $\tau_{\mathrm{d}} / \tau_{\mathrm{r}}$ ratios $(-:$ ratio $=10,--$ : ratio $=30,-\cdot$ : ratio $=100, \cdots:$ ratio $=300)$.

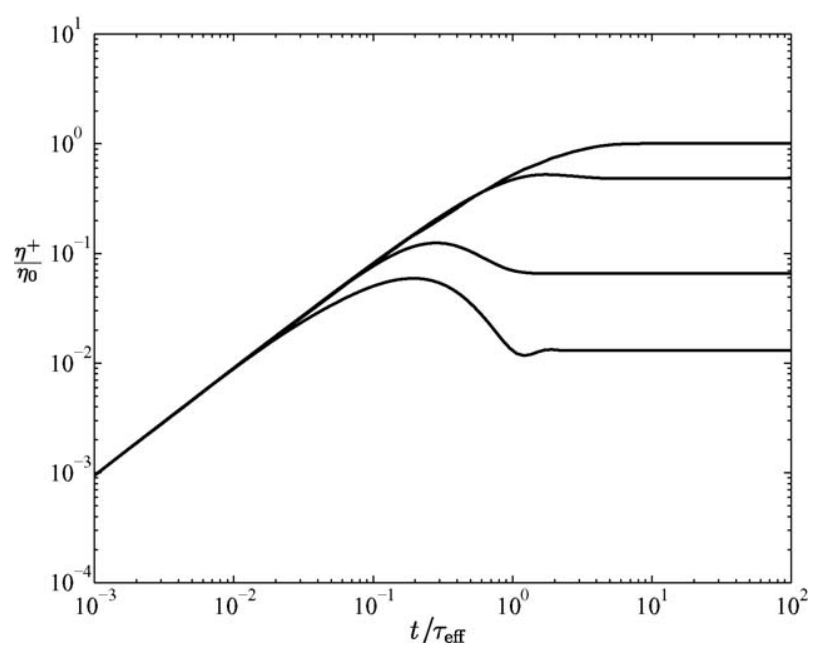

Fig. 3. Transient shear viscosity for four different Deborah numbers. From the top, the respective Deborah numbers are $0.01,1,10$ and 100. Finite extensibility has been neglected and $\tau_{\mathrm{d}} / \tau_{\mathrm{r}}=30$.

prevent the shear stress maximum from ocurring. We nevertheless believe that a better understanding of convective constraint release, especially in reversing flows, could lead to improved formulations where such modifications would not be necessary.

Fig. 3 shows the transient shear viscosity for various shear rates for $\tau_{\mathrm{d}} / \tau_{\mathrm{r}}=100$ and no finite exensibility effects. We observe that as the shear rate increases, an overshoot appears in the viscosity curve due to the transient stretching of the chain. At high shear rates the model also predicts a slight undershoot following the undershoot. In a following section we will see that these over- and under-shoots are observed experimentally and can be predicted quantitatively.

In Fig. 4, we show the effects of finite extensibility on the predictions of the steady state shear stress. The parameters are identical to those used for Fig. 2 except for the finite extensibility parameter which we set to $b=100$. Accounting for finite extensibility only changes the results at high shear rates, where it leads to reduced levels of stress.

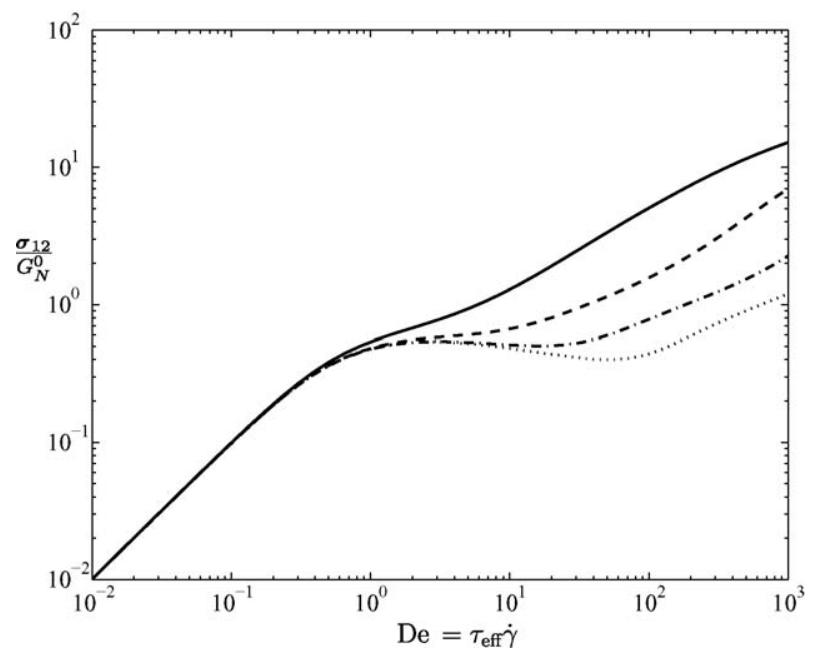

Fig. 4. Steady state shear stress as a function of a Deborah number. The four curves correspond to different $\tau_{\mathrm{d}} / \tau_{\mathrm{r}}$ ratios (-: ratio $=10,--$ : ratio $=30$, - : ratio $=100, \cdots:$ ratio $=300)$. The parameter $b$ is set to $b=100$. 


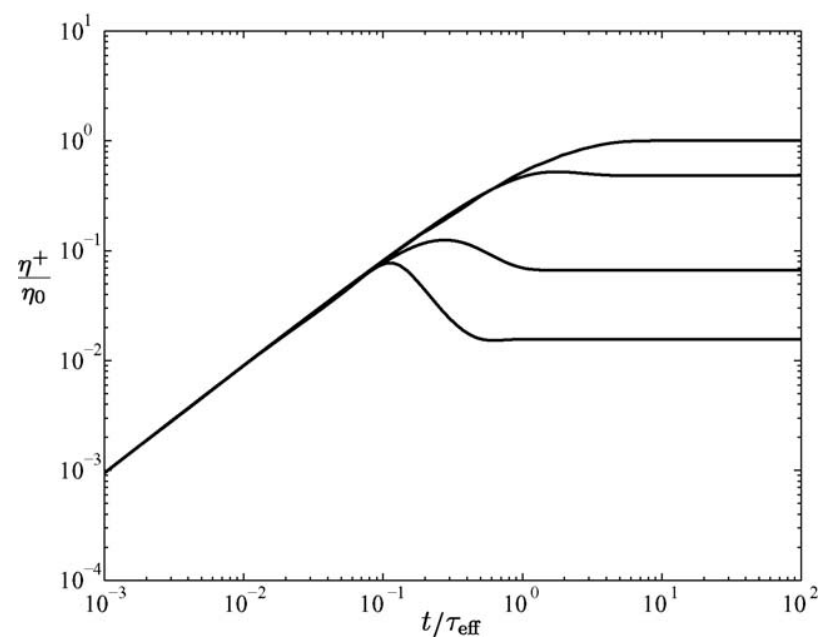

Fig. 5. Transient shear viscosity for four different Deborah numbers. From the top, the respective Deborah numbers are $0.01,1,10$ and 100 . The finite extensibility parameter $b$ is set to 100 and $\tau_{\mathrm{d}} / \tau_{\mathrm{r}}=30$.

In Fig. 5, we show the transient shear viscosity for different Deborah numbers. The ratio $\tau_{\mathrm{d}} / \tau_{\mathrm{r}}$ is set to 30 while the finite extensibility parameter is set to 100 . Accounting for finite extensibility can eventually lead, in the transient regime of very fast flows, to stress levels corresponding to more than affine deformations.

\section{Predictions in uniaxial extension}

As seen in recent publications $[35,3,36,19]$, there is a growing interest in measuring, predicting and understanding extensional flows of entangled systems. Fig. 6 shows the steady state Trouton ratio as predicted by the CRAFT model for different values of the finite extensibility parameter $b$ and a ratio $\tau_{\mathrm{d}} / \tau_{\mathrm{r}}$ of 100 . As the extension rate increases we clearly see four different regimes.

(1) The first regime corresponds to slow flows, where the material can relax sufficiently fast in order to maintain its equilibrium structure. Its response is therefore linear and the Trouton ratio is constant. As the flow rate increases, the microstructure begins to align in the flow direction. At the onset of this phenomenon, the additional stress, only due to orientation, actually grows faster that the flow rate. This is why one can observe a shallow maximum of the Trouton ratio when $\dot{\epsilon} \tau_{\mathrm{d}}$ is of order unity.

(2) For increasing flow rates, reptation cannot prevent the microstructure from reaching a full orientation, but stretch relaxation is still fast enough to maintain the chains unstretched. As the stress saturates, we observe a decrease of the Trouton ratio. In this regime, the slope of the curve can reach a value of -1 , if the reptation and stretch relaxation times are well separated. Even for a monodisperse system, the transition to this regime can actually be quite slow as our model exhibits the full spectrum of reptation coupled with constraint release.

(3) When the flow is fast enough to stretch the chain, the extensional stress grows again beyond the value corresponding to

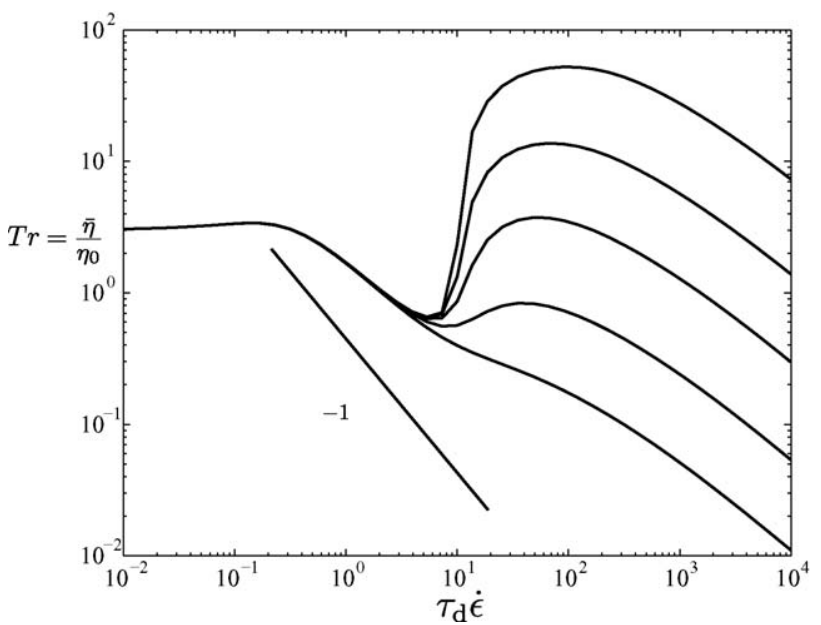

Fig. 6. Steady state Trouton ratio for $\tau_{\mathrm{d}} / \tau_{\mathrm{r}}=30$ and different values of the finite extensibility parameter $b$. From the top, the respective values of $b$ are 1000, 300, 100,30 and 10.

full orientation of the microstructure in the flow direction. The stress growth is stopped when the chain reaches its full extension, and that is why this growth is not seen at all if the finite extensibility parameter $b$ is small enough.

(4) In the fourth regime, the Trouton ratio is a decreasing function of the extension rate. In this regime constraint release is the dominant mechanism and, as the chain is fully stretched, it has to retract as fast as it is stretched by the flow in order to remain below its maximum length. The induced rates of CCR are therefore of the order of the extension rate, which explains the decreasing Trouton ratio. The exact shape of the curve actually depends on the non-linear expressions involved in the implementation of finite extensibility.

In Fig. 6, we see that for low values of the parameter $b$, the third regime is suppressed and the extensional viscosity is a monotonously decreasing function. Indeed, as the extensibility of the chains is very small they are almost instantaneously stretched to their maximum length once the flow rate is high enough. This instantaneous transition from unstretched to stretched corresponds to the instantaneous transition from the second to the fourth regime.

\section{The CRAFT constitutive equation for polydisperse systems}

So far, we have focused on monodisperse systems, where all molecules have the same mass. In this section, we present the extension of the CRAFT constitutive equation to the polydisperse case. Going from the monodisperse to the polydisperse case with the CRAFT model is actually very similar to what we did for linear visco-elasticity: the key issue is to find a consistent expression for the rate of constraint release $f_{\text {ccr }}$ which couples the relaxation of all masses.

Let us consider a polymeric system with $N$ different molecular masses $M^{(i)}$, each of them having a volume fraction $\phi^{(i)}$. The polydisperse CRAFT constitutive equation assumes that all masses relax independently except for the constraint release 
term. Indeed, as the chains all relax within the same environment, the rate of relaxation $f_{\text {ccr }}$ must be the same for all masses and must be computed from the rate of relaxation of all masses. Furthermore, a polydisperse environment yields a broad spectrum of relaxation times, and we cannot further assume a single relaxation time $\tau_{\mathrm{cr}}$ for thermal constraint release but a spectrum of $N_{\mathrm{CR}}$ characteristic times $\tau_{j}$. These times $\tau_{j}$ and their respective weights $w_{j}$ are computed as described in Section 2 and ensure the correct linear viscoelastic limit of the model.

The polydisperse CRAFT constitutive is expressed as a set of $N$ times $N_{\text {CR }}$ partial differential equations (one per mass fraction and one per thermal constraint release mode) which are coupled only through the single term of convective constraint release. The variables of the model are the set of conformation tensors $\mathbf{c}^{(i)}(t, s)$, which describes the conformation of all masses along the primitive path. The equations for the polydisperse CRAFT model read:

$$
\begin{aligned}
\nabla^{(i, j)}(t, s)= & \frac{\partial}{\partial s}\left(\alpha_{\mathrm{d}}^{f(i)}(s) \frac{\partial}{\partial s}\left(f^{(i, j)} \mathbf{c}^{(i, j)}\right)\right) \\
& +\alpha_{\mathrm{r}}^{(i)} \frac{\mathbf{c}^{(i, j)}}{\operatorname{tr} \mathbf{c}^{(i, j)}} \frac{\partial^{2}}{\partial s^{2}}\left(f^{(i, j)} \operatorname{tr} \mathbf{c}^{(i, j)}\right) \\
& +\frac{\alpha_{\mathrm{r}}^{(i)}}{2} \frac{\partial\left(f^{(i, j)} \operatorname{tr} \mathbf{c}^{(i, j)}\right)}{\partial s} \frac{\partial}{\partial s}\left(\frac{\mathbf{c}^{(i, j)}}{\operatorname{tr} \mathbf{c}^{(i, j)}}\right) \\
& -\left(\frac{1}{\tau_{j}}+f_{\mathrm{ccr}}\right) \frac{3}{\operatorname{tr} \mathbf{c}^{(i, j)}}\left(f^{(i, j)} \mathbf{c}^{(i, j)}-\delta\right),
\end{aligned}
$$

with,

$\mathbf{c}^{(i, j)}(t, \pm 1)=\delta$

$f_{\mathrm{ccr}}=-\frac{\left.\sum_{i, j} w_{j} \phi^{(i)} \int_{-1}^{1}\left(\frac{D a\left(\mathbf{c}^{(i, j)}\right)}{D t}\right)\right|_{\text {stretch }} \mathrm{d} s}{\sum_{i, j} w_{j} \phi^{(i)} \int_{-1}^{1} a\left(\mathbf{c}^{(i, j)}\right) \mathrm{d} s}$,

$f(\mathbf{c})=\frac{b-3}{b-\operatorname{tr} \mathbf{c}}$,

$a(\mathbf{c})=G_{N}^{0}\left(-(b-3) \ln \left(1-\frac{\operatorname{tr} \mathbf{c}}{b}\right)-\ln \operatorname{det}(\mathbf{c})\right)$,

$\boldsymbol{\tau}_{p}=G_{N}^{0} \sum_{i=1}^{N} \sum_{j=1}^{N_{\mathrm{CR}}} w_{j} \phi^{(i)} \frac{1}{2} \int_{-1}^{1}(f \mathbf{c}-\boldsymbol{\delta}) \mathrm{d} s$.

In the linear limit, this set of equations actually reduces to the linear model recalled in Section 2. In the non-linear regime, the CRAFT constitutive equation provides a mixing rule where the coupling mechanism gradually switches from thermal to convective constraint release. The definition of the rate of convective constraint release $f_{\text {ccr }}(39)$ is a natural extension of the monodisperse case that still ensures a positive instantaneous relaxation time.

\section{Comparison with experimental data}

In two successive publications, Pattamaprom et al. [2] and Ye et al. [3] presented a complete set of experimental data for mono and bi-disperse entangled polystyrene solutions. The features of
Table 1

Description of the six entangled polystyrene solutions S1-S6 [2]

\begin{tabular}{ll}
\hline Name & Features \\
\hline S1 & 7 vol. $\%$ PS $M_{\mathrm{w}}=2.9 \times 10^{6} \mathrm{Da}, M_{\mathrm{w}} / M_{n}=1.09$ \\
S6 & 7 vol. $\%$ PS $M_{\mathrm{w}}=8.4 \times 10^{6} \mathrm{Da}, M_{\mathrm{w}} / M_{n}=1.17$ \\
S2 & $80 \% \mathrm{~S} 1,20 \% \mathrm{~S} 6$ \\
S3 & $60 \% \mathrm{~S} 1,40 \% \mathrm{~S} 6$ \\
S4 & $40 \% \mathrm{~S} 1,60 \% \mathrm{~S} 6$ \\
S5 & $20 \% \mathrm{~S} 1,80 \% \mathrm{~S} 6$ \\
\hline
\end{tabular}

Table 2

Parameters of the CRAFT model used for comparison with solutions S1-S6 at $40{ }^{\circ} \mathrm{C}$

\begin{tabular}{ll}
\hline$K_{\mathrm{d}}\left(\mathrm{s} \mathrm{Da}^{-3}\right)$ & $2.43 \times 10^{-19}$ \\
$K_{\mathrm{r}}\left(\mathrm{s} \mathrm{Da}^{-2}\right)$ & $1.0 \times 10^{-14}$ \\
$K_{\mathrm{f}}$ & 1 \\
$G_{N}^{0}(\mathrm{~Pa})$ & 465 \\
$\gamma$ & 1.0 \\
$b$ & 942 \\
\hline
\end{tabular}

the six solutions, named S1 to S6 are reported in Table 1. From the linear viscoelastic moduli of S6 shown in Fig. 11, one sees that it contains a significant fraction of smaller masses. We will nevertheless assume that S6 is monodisperse and consider S2S5 as strictly bi-disperse samples.

For all solutions, the authors reported the linear viscoelastic moduli, the steady shear viscosity and first normal stress difference as well as the steady uniaxial extensional stresses. For some solutions, transient shear and extensional data were also reported. As different reference temperatures were used for the experiments presented in [2] and [3], we shifted the timescales of the extensional stress measurements to have a reference temperature of $40^{\circ} \mathrm{C}$.

This set of data allows us to specifically test the various components of the CRAFT constitutive equation. In a first step, we evaluate the ability of the CRAFT model to fit the experimental results for the monodisperse system $\mathrm{S} 1$. Then we validate the ability to predict the response for the other monodisperse system S6 through the two scaling laws of Eqs. (3) and (35). Finally we test the mixing rule in the non-linear regime on the solutions S2-S5.

The procedure we used to adjust the parameters of the CRAFT model is the following:

- We adjusted the parameters $K_{\mathrm{d}}, G_{N}^{0}$ and $\gamma$ in order to quantitatively predict the low and intermediate frequencies of the viscoelastic moduli of S1. In this process, we assumed $K_{\mathrm{f}}=1$.

- Using the transient shear viscosity data for $\mathrm{S} 1$, we adjusted the parameter $K_{\mathrm{r}}$. Only the data for the highest extension rate was used.

- The finite extensibility parameter, derived on a microstructural basis, was taken from [3].

Table 2 summarizes the values of the parameters.

In Fig. 7, we see the comparison between the experimental viscoelastic moduli of S1, and the predictions of the constitutive equation. At high frequencies, a Rouse spectrum contribution was added to the predictions. The Rouse time for the linear re- 


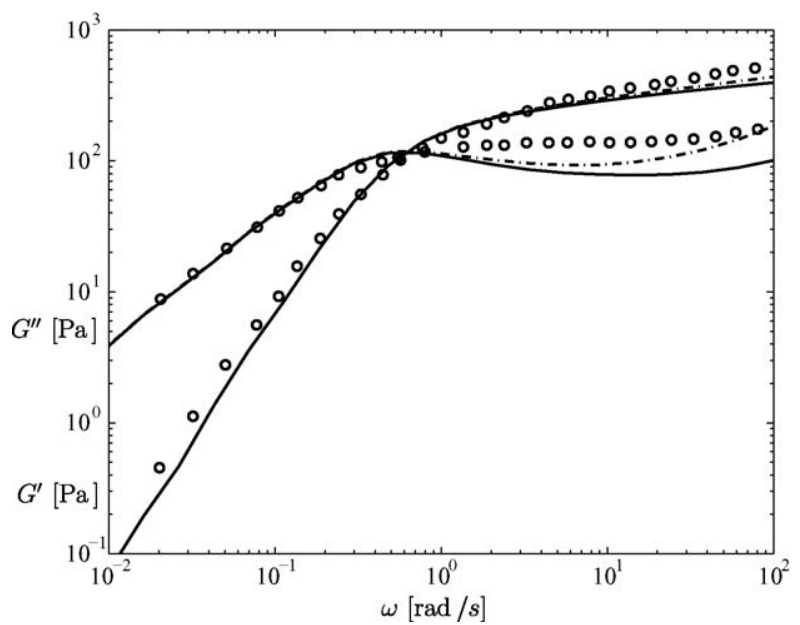

Fig. 7. Linear viscoelastic moduli for S1. Comparison between experimental results [2](०) and the CRAFT model $(-)$. The dashed lines are the predictions of the model for $K_{\mathrm{r}}=3.4 \times 10^{-12}\left(\mathrm{~s} \mathrm{Da}^{-2}\right)$.

sponse was computed as $\tau_{\mathrm{r}}=K_{\mathrm{r}} M^{2}$ and is therefore identical to the characteristic time of stretch relaxation. From the comparison with the experimental data, it seems that a longer Rouse time should have been used in order to have quantitative predictions in the whole frequency range.

In Fig. 8, we report the experimental transient shear viscosity $\eta^{+}$for $\mathrm{S} 1$, which we used for adjusting the stretch relaxation time of the model, together with the model predictions. We see that, with the appropriate stretch relaxation time, the CRAFT model predicts the steady state values and the transient behaviour. At high shear rates, the CRAFT model quantitatively predicts the large overshoot in viscosity followed by a shallow undershoot. At intermediate shear rates however, the model fails to quantitatively predict the overshoot in shear viscosity: stress built up during the overshoot relaxes too quickly. This failure is actually consistent with our observations for the linear response. In

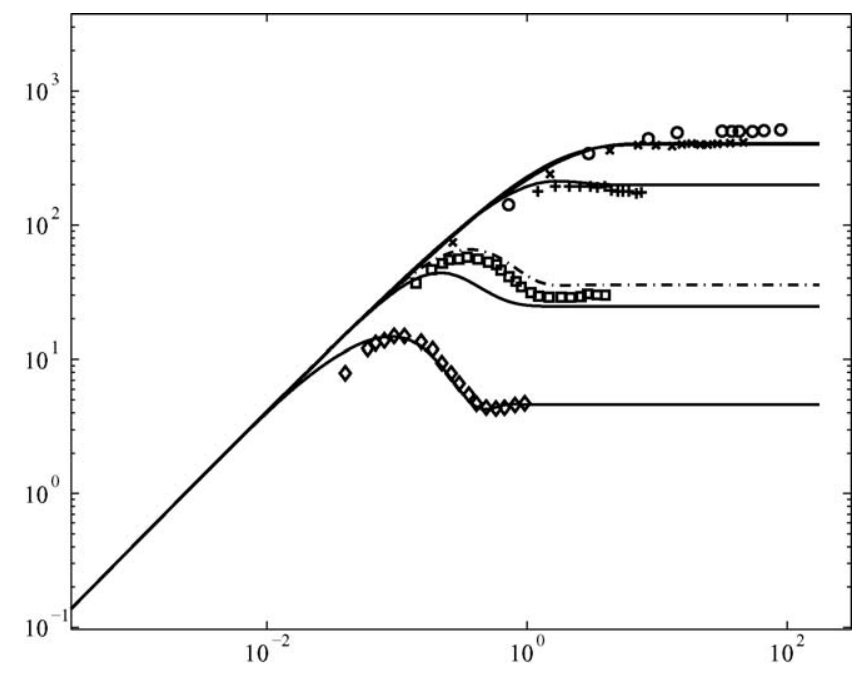

Fig. 8. Transient shear viscosity for S1. Comparison between experimental results [2] (symbols) and the CRAFT model (-). From top to bottom, the curves correspond to the following flow rates: $0.01\left(\mathrm{~s}^{-1}\right), 0.1\left(\mathrm{~s}^{-1}\right), 1\left(\mathrm{~s}^{-1}\right), 10\left(\mathrm{~s}^{-1}\right)$ and $100\left(\mathrm{~s}^{-1}\right)$. The dashed lines is the prediction of the model for $K_{\mathrm{r}}=3.4 \times 10^{-12}$ $\left(\mathrm{s} \mathrm{Da}^{-2}\right)$, at a flow rate of $10\left(\mathrm{~s}^{-1}\right)$.

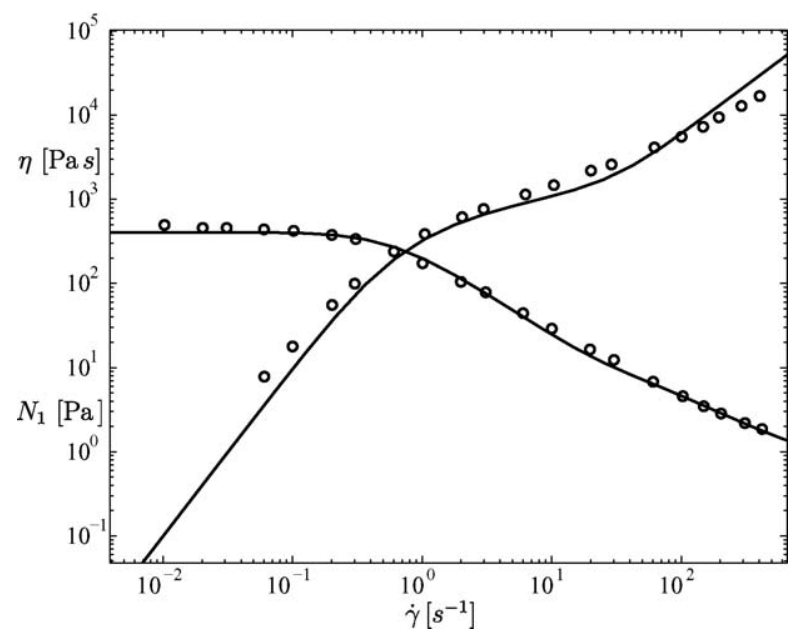

Fig. 9. Steady state shear viscosity $\eta$ and first normal stress difference $N_{1}$ for S1. Comparison between experimental results [2] (०) and the CRAFT model $(-)$.

Figs. 7 and 8, the dashed lines are the predictions of the model with the Rouse time increased by a factor 3.4. We see that this yelds simultaneously better quantitative predictions both for the linear moduli and the transient shear viscosity at low deformation rates.

This closes the first step of parameters adjustment: the parameters are now frozen and we can examine the predictions of the models in shear for S1 and S6, and in shear and extension for the blends S2-S5.

As foreseen in Figs. 8 and 9 shows the excellent agreement of the steady shear viscosity of S1 with the model predictions. The steady state first normal stress difference is predicted quantitatively as well. The main difference between the data and the predictions is the "kink" one observes on the predicted curves at shear rates for which the chain becomes significantly stretched. These are not observed experimentally and suggest that the model predicts too much chain stretch in shear.

In Fig. 10, the experimental steady extensional viscosity is compared to the model predictions. The rise of the

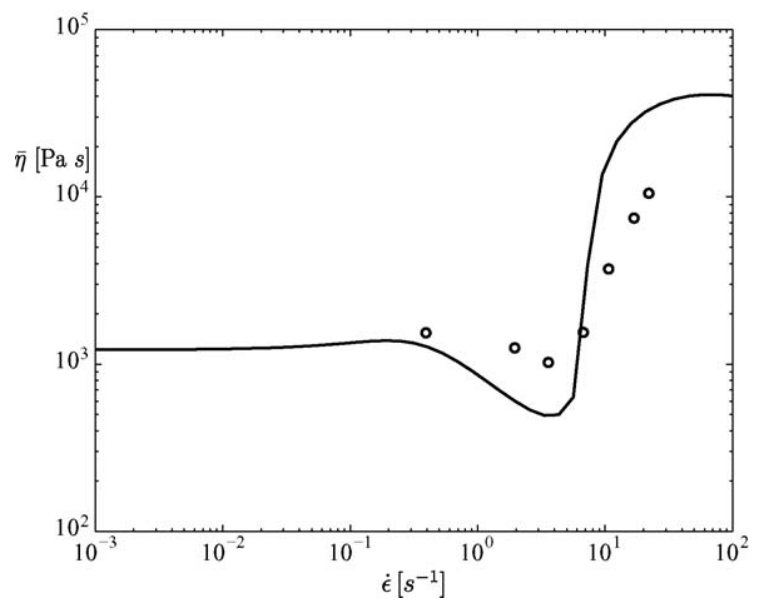

Fig. 10. Steady extensional viscosity for S1. Comparison between experimental results [3] (o) and the CRAFT model (-). 


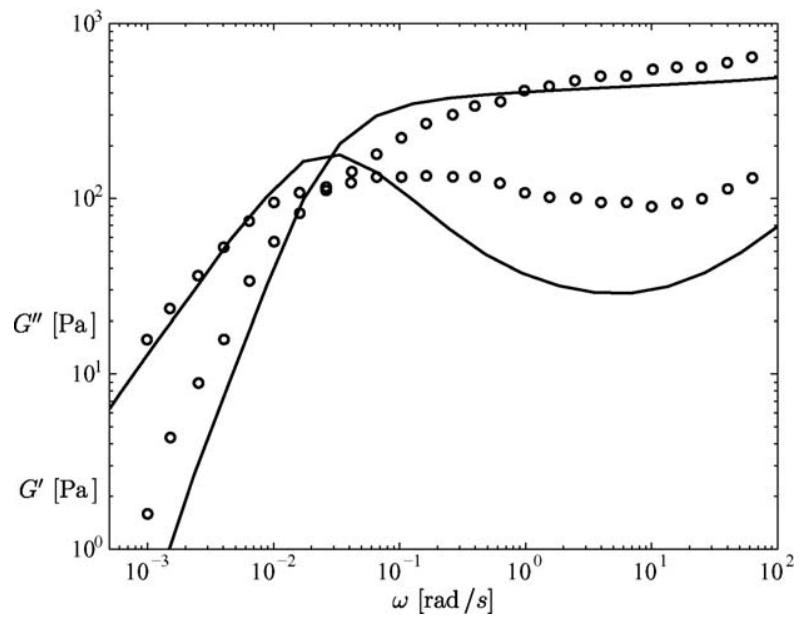

Fig. 11. Linear viscoelastic moduli for S6. Comparison between experimental results [2] (o) and the CRAFT model (-).

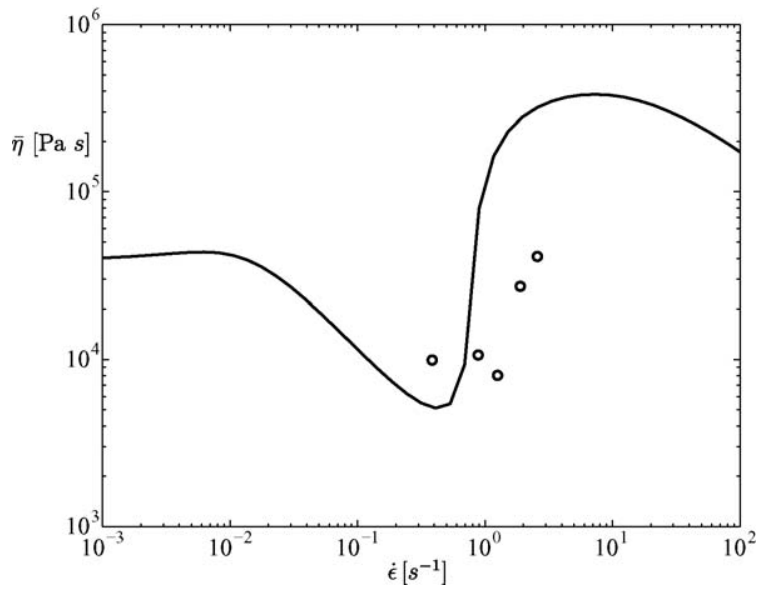

Fig. 12. Steady extensional viscosity for S6. Comparison between experimental results [3] (०) and the CRAFT model (-).

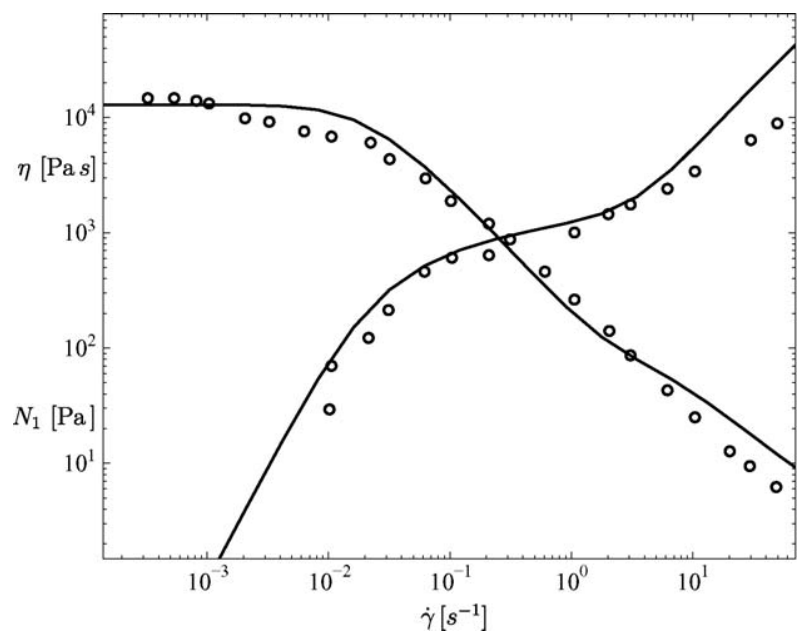

Fig. 13. Steady state shear viscosity $\eta$ and first normal stress difference $N_{1}$ for S6. Comparison between experimental results [2] (०) and the CRAFT model $(-)$. extensional viscosity due to the onset of chain stretch is well described, qualitatively and quantitatively, which tends to confirm the value we selected for the Rouse time. The viscosity decay observed at lower extension rates is overestimated by the model. Similar predictions have been obtained by Bhattacharjee et al. [35] in comparing similar entangled solutions with the predictions of different constitutive models. Further modelling efforts need to be spent to describe these deviations from the theoretical -1 slope. The fourth regime (see Section 6) of the extensional viscosity is unfortunately out of the range of the experiments and the validity of the CRAFT predictions at high extension rates cannot be assessed.

Fig. 11 offers the same comparison as Fig. 7 but for the solution S6. Looking at the experimental loss modulus for intermediate frequencies clearly shows that the sample is not monodisperse as we are assuming. Nevertheless, we are able to correctly predict the low frequency range of the loss modulus, which ensures good predictions of the zero-shear viscosity $\eta_{0}$. As for solution S1, the Rouse time of the model is again too high to describe well the high frequency regime of $G^{\prime}$ and $G^{\prime \prime}$.

In Fig. 12, we show that the scaling law for $\alpha_{\mathrm{r}}$ allows the CRAFT model to make good predictions of the extensional viscosity of solution S6. The onset of chain stretch is well predicted, but the sparsity of the data prevents any further analysis. The presence of shorter chains in the sample is likely to explain the somewhat delayed hardening observed in the experimental data.

In Fig. 13, we see that the model correctly predicts the steady shear viscosity and first normal stress difference of S6 over a wide range of shear rates. Discrepancies between predictions and experiments only occur at high shear rates, where the model predicts that the flow starts stretching the chains, leading to a change of slope of the curves. The kink in the predictions occurring at high shear rates is more visible for S6 than it is for S1, as the increase in molecular weight yields a greater separation between $\tau_{\mathrm{d}}$ and $\tau_{\mathrm{r}}$.

Fig. 14 shows the predictions of the steady shear viscosity and first normal stress difference for the solutions S2-S5. One should remember that the predictions for solutions S2 to S5 did not require any additional material parameter. From a global point of view, one can say that the agreement between predictions and experiments is quite good, but several comments arise from a closer look at the curves.

- The model is able to give a reasonable prediction of the zero shear rate viscosity $\eta_{0}$ for all solutions. This linear limit can be expected from the linear theory behind the CRAFT model.

- For CCR dominated shear rates, the predictions of the CRAFT are in very good agreement with experiments. The non-linear mixing rule coming from our generalized convective constraint release seems quite efficient in this regime.

- As for the monodisperse samples, the CRAFT model predicts a transition to a "stretched regime" at high shear rates, which is not seen in the experiments. 

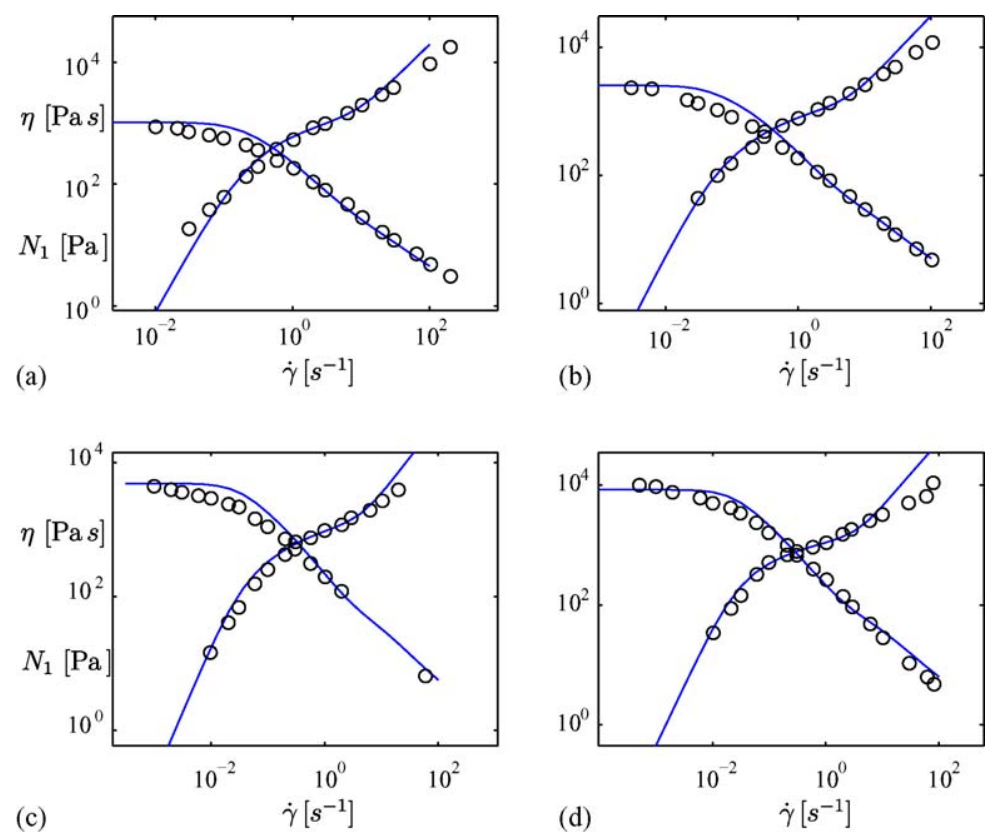

Fig. 14. Steady state shear viscosity $\eta$ and first normal stress difference $N_{1}$ for S2 (a), S3 (b), S4 (c) and S5 (d). Comparison between experimental results [2] (o) and the CRAFT model (-)
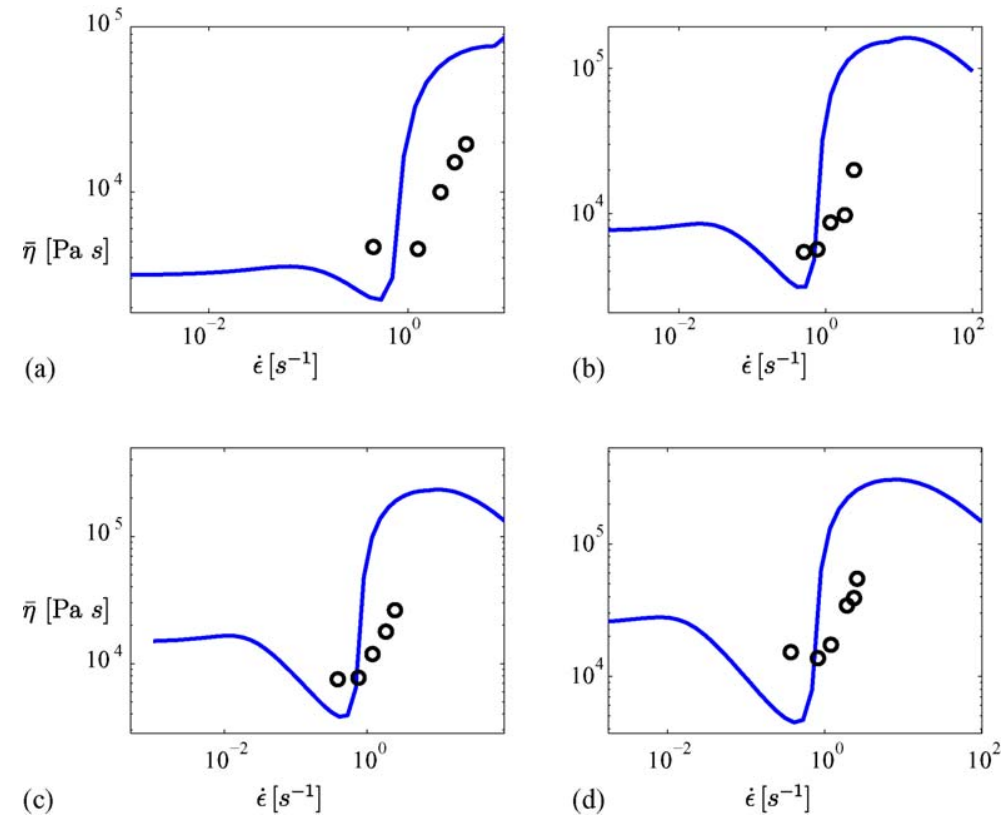

Fig. 15. Steady extensional viscosity for S2 (a), S3 (b), S4 (c) and S5 (d). Comparison between experimental results [3] (o) and the CRAFT model (-).
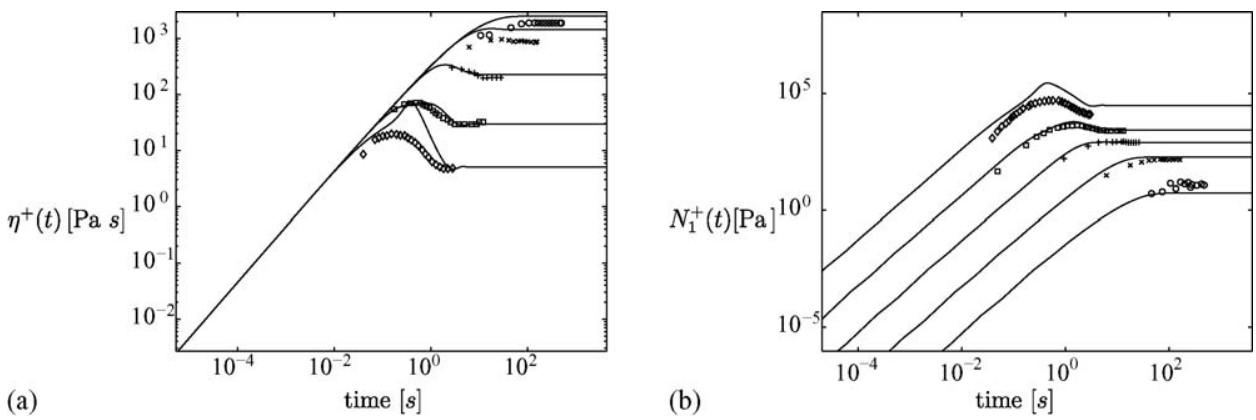

Fig. 16. Transient shear viscosity (a) and transient first normal stress difference for S3. Comparison between experimental results [2] (symbols) and the CRAFT model (-). From top to bottom (a) and bottom to top (b), the curves correspond to the following flow rates: $0.01\left(\mathrm{~s}^{-1}\right), 0.1\left(\mathrm{~s}^{-1}\right), 1\left(\mathrm{~s}^{-1}\right), 10\left(\mathrm{~s}^{-1}\right)$ and $100\left(\mathrm{~s}^{-1}\right)$. 
Fig. 15 shows the predictions of the steady extensional viscosity together with experimental measurements for solutions S2-S5. Because only few experimental points are available, we can only observe that the model is able to predict the extensional viscosity growth at about the right extension rate. Additionally, we still observe a too strong extensional thinning of the model. The experimental extension rates are too small in order to validate the behaviour of the CRAFT model in the last regime of the viscosity curve.

In Fig. 16, we show both the transient shear viscosity and first normal stress difference for S3. The ability of the CRAFT model to predict the transient response of bi-disperse samples actually is a strong test for its mixing rule. We see that for low and medium shear rates, the predictions are in excellent agreement with the data. At high shear rates, the occurrence of high levels of stretch ruins the transient predictions.

\section{Conclusions}

We have proposed a new tube-based constitutive equation named CRAFT for polydisperse linear entangled polymers. The model has the form of a set of coupled partial differential equations for a configuration tensor defined along the primitive chain coordinate.

In addition to reptation, contour-length fluctuations and thermal constraint release, CRAFT accounts for the coupled relaxation of stretch and orientation along the primitive chain. convective constraint release (CCR) appears naturally in the model, as a non-linear correction to thermal constraint release.

The description of polydisperse systems with the CRAFT model requires no additional material parameter and the coupling between the different masses is provided by constraint release effects. In the linear regime the CRAFT model reduces to a simple theory [1] capable of quantitative predictions, even for polydisperse systems. The deep connection of the CRAFT model with a linear theory allows the identification of most of the parameters on linear viscoelastic data only.

The preliminary comparison of the CRAFT predictions with experimental data is very promising. The model is able to correctly predict both the linear and non-linear rheology of linear polymers with a single set of parameters. On mono- and bi-disperse entangled polystyrene solutions, we have show that the CRAFT model is able to correctly predict the steady shear viscosity and first normal stress difference over a wide range of shear rates. Additionally, the onset of extensional strain hardening is well predicted for steady state flows. Transient experiments can be quantitatively predicted as well. The lesser quality of the predictions at high shear rates and the excessive extensional thinning of the model tend to indicate that additional stretch relaxation mechanisms, possibly related to CCR should be accounted for.

\section{Acknowledgments}

This work is supported by the "Action de Recherche Concertée" program of the Communauté française de Belgique and by "BASF Aktiengesellschaft". The authors wish to thank G. Marrucci and G. Ianniruberto for fruitful discussions.

\section{Appendix A. On the rate of CCR}

In this appendix, we prove that under certain conditions and assumptions, the rate of convective constraint release defined through Eq. (26) is always positive. For clarity, we will restrict ourselves to the monodisperse case.

From the definition of the tensor $\mathbf{c}(t, s)$ we see that its equilibrium value is the identity tensor. Furthermore, the evolution equation for $\mathbf{c}(t, s)$, is such that $\mathbf{c}(t, s)$ always remains symmetri$\mathrm{cal}$. We now assume that the tensor $\mathbf{c}(t, s)$ always remains strictly positive definite. We do not prove this assumption but if, for an arbitrary flow history, one of the eigenvalues of $\mathbf{c}(t, s)$ was to become negative it would have to become null, as a result of continuity. As the logarithm of the determinant of $\mathbf{c}(t, s)$ enters the evolution equation, we see that the differential equation would not be defined in such case.

When finite extensibility is not accounted for, $a(\mathbf{c})$ is defined as:

$a(\mathbf{c})=\operatorname{tr}(\mathbf{c})-\ln \operatorname{det}(\mathbf{c})$,

and is equal to 3 at equilibrium. The gradient of $a(\mathbf{c})$ with respect to $\mathbf{c}$ is:

$\frac{\partial a}{\partial \mathbf{c}}=\left(\boldsymbol{\delta}-\mathbf{c}^{-1}\right)$.

The function $a(\mathbf{c})$ is strictly positive when $\mathbf{c}$ is positive definite. This can be proved by noticing that the gradient of $a(\mathbf{c})$ with respect to $\mathbf{c}$ only vanishes at equilibrium and that the Hessian is positive definite as well. The denominator on the right hand side of Eq. (26) is therefore always strictly greater than zero. Let us now prove that the numerator (without the minus sign) is strictly negative.

The expression inside the integral of the numerator of Eq. (26) can be written as follows:

$$
\begin{aligned}
\left.\frac{D}{D t} a(\mathbf{c}(t, s))\right|_{\text {stretch }} & =\alpha_{\mathrm{r}} \frac{\partial^{2}}{\partial s^{2}}(\operatorname{tr} \mathbf{c}) \frac{\mathbf{c}}{\operatorname{tr} \mathbf{c}}: \frac{\partial a}{\partial \mathbf{c}} \\
& =\alpha_{\mathrm{r}} \frac{\partial^{2}}{\partial s^{2}}(\operatorname{tr} \mathbf{c}) \frac{\mathbf{c}}{\operatorname{tr} \mathbf{c}}:\left(\boldsymbol{\delta}-\mathbf{c}^{-1}\right) \\
& =\alpha_{\mathrm{r}} \frac{\partial^{2}}{\partial s^{2}}(\operatorname{tr} \mathbf{c})\left(1-\frac{3}{\operatorname{tr} \mathbf{c}}\right) .
\end{aligned}
$$

The numerator of Eq. (26) is therefore equal to:

$$
\int_{-1}^{1} \alpha_{\mathrm{r}} \frac{\partial^{2}}{\partial s^{2}}(\operatorname{tr} \mathbf{c})\left(1-\frac{3}{\operatorname{tr} \mathbf{c}}\right) \mathrm{d} s
$$

We will now conclude the proof by showing that the previous expression is always strictly negative. 
Let $g(s)$ be a continuous and sufficiently smooth function on the interval [-11]. If $g(s)$ is strictly positive and is such that $g(1)=g(-1)=3$, we have the following result:

$\int_{-1}^{1} \frac{\partial^{2}}{\partial s^{2}} g\left(s^{\prime}\right)\left(1-\frac{3}{g\left(s^{\prime}\right)}\right) \mathrm{d} s^{\prime} \leq 0$.

The proof of this inequality only requires basic calculus and one integration by parts.

$$
\begin{aligned}
& \int_{-1}^{1} \frac{\partial^{2}}{\partial s^{2}} g\left(s^{\prime}\right)\left(1-\frac{3}{g\left(s^{\prime}\right)}\right) \mathrm{d} s^{\prime} \\
& \quad=\int_{-1}^{1} \frac{\partial^{2}}{\partial s^{2}} g\left(s^{\prime}\right) \mathrm{d} s^{\prime}-\int_{-1}^{1} \frac{3}{g\left(s^{\prime}\right)} \frac{\partial^{2}}{\partial s^{2}} g\left(s^{\prime}\right) \mathrm{d} s^{\prime} .
\end{aligned}
$$

The first term is simply integrated, while the second is integrated by parts:

$$
\begin{aligned}
& =\left[\frac{\partial}{\partial s} g(s)\right]_{-1}^{1}-\left[\frac{3}{g(s)} \frac{\partial}{\partial s} g(s)\right]_{-1}^{1}-\int_{-1}^{1} \frac{3}{g\left(s^{\prime}\right)^{2}}\left(\frac{\partial}{\partial s} g\left(s^{\prime}\right)\right)^{2} \\
& \mathrm{~d} s^{\prime}=-\int_{-1}^{1} \underbrace{\frac{3}{g\left(s^{\prime}\right)^{2}}\left(\frac{\partial}{\partial s} g\left(s^{\prime}\right)\right)^{2}}_{\geq 0} \mathrm{~d} s^{\prime} \leq 0 .
\end{aligned}
$$

The two first terms of Eq. (A.2) cancel each other as $g(-1)=$ $g(1)=3$, while the last term is non-negative. This concludes the proof that our definition of the rate of CCR always yields a non-negative value, even when the flow tends to drive the chain below its equilibrium length.

\section{References}

[1] A. Leygue, C. Bailly, R. Keunings, A differential tube-based model for predicting the linear viscoelastic moduli of polydisperse entangled linear polymers, J. Non Newton. Fluid Mech. 133 (2006) 2834.

[2] C. Pattamaprom, R.G. Larson, Constraint release effects in monodisperse and bidisperse polystyrenes in fast transient shearing flows, Macromolecules 34 (2001) 5229-5237.

[3] X. Ye, R.G. Larson, C.J. Pattamaprom, T. Shridar, Extensional properties of monodisperse and bidisperse polystyrene solutions, J. Rheol. 47 (2) (2003) 443-468.

[4] P.G. De Gennes, Reptation of a polymer chain in the presence of fixed obstacles, J. Chem. Phys. 55 (1971) 572-579.

[5] M. Doi, S.F. Edwards, The Theory of Polymer Dynamics, Oxford University Press, 1986.

[6] E. van Ruymbeke, R. Keunings, V. Stéphenne, A. Hagenaars, C. Bailly, Evaluation of reptation models for predicting the linear viscoelastic properties of entangled linear polymers, Macromolecules 35 (7) (2002) 2689 2699.

[7] A.E. Likhtman, T.C.B. McLeish, Quantative theory for linear dynamics of linear entangled polymers, Macromolecules 35 (2002) 63326343.

[8] S.T. Milner, T.C.B. McLeish, Reptation and contour-length fluctuations in melts of linear polymers, Phys. Rev. Lett. 81 (3) (1998) 725-728.

[9] C.C. Hua, J.D. Schieber, D.C. Venerus, Segment connectivity, chain-length breathing, segmental stretch, and constraint release in reptation models. i. Theory and single-step strain predictions, J. Chem. Phys. 109 (22) (1998) 10018-10032.
[10] Y. Masubuchi, J. Takimoto, K. Koyama, G. Ianniruberto, G. Marrucci, F. Grecco, Brownian simulations of a network of reptating primitive chains, J. Chem. Phys. 115 (9) (2001) 4387-4394.

[11] J. Des Cloizeaux, Relaxation of entangled polymers in melts, Macromolecules 23 (17) (1990) 3992-4006.

[12] J. Des Cloizeaux, Relaxation and viscosity anomaly of melts made of long entangled polymers, Macromolecules 23 (21) (1990) 46784687.

[13] R.S. Graham, A.E. Likhtman, T.C.B. McLeish, S.T. Milner, Microscopic theory of linear entangled polymer chains under rapid deformation including chain stretch and convective constraint release, J. Rheol. 47 (5) (2003) 1171-1200.

[14] G. Marrucci, Relaxation by reptation and tube enlargement: a model for polydisperse polymers, J. Polym. Sci., Polym. Phys. 23 (1985) 159177.

[15] J.L. Viovy, M. Rubinstein, R.H. Colby, Constraint release in polymer melts: tube reorganization versus tube dilation, Macromolecules 24 (12) (1991) 3587-3596.

[16] E. van Ruymbeke, R. Keunings, C. Bailly, Prediction of linear viscoelastic properties for polydisperse mixtures of entangled star and linear polymers: modified tube-based model and comparison with experimental results, J. Non Newton. Fluid Mech. 128 (1) (2005) 7-22.

[17] C. Tsenoglou, Viscoelasticity of binary polymer blends, ACS Polym. Prepr. 28 (1987) 185-186.

[18] J. Des Cloizeaux, Double reptation vs. simple reptation in polymer melts, J. Europhys. Lett. 5 (1988) 437-442.

[19] G. Marrucci, G. Ianniruberto, Interchain pressure effect in extensional flows of entangled polymer melts, Macromolecules 37 (2004) 39343942 .

[20] G. Marrucci, N. Grizzuti, Fast flows of concentrated polymers-predictions of the tube model on chain stretching, Gazz. Chim. Ital. 118 (1988) 179185.

[21] G. Marrucci, Dynamics of entanglements: a non-linear model consistent with the Cox-Merz rule, J. Non Newton. Fluid Mech. 62 (1996) 279289.

[22] G. Ianniruberto, G. Marrucci, On compatibility of the Cox-Merz rule with the model of Doi and Edwards, J. Non Newton. Fluid Mech. 65 (2/3) (1996) 241-246.

[23] G. Marrucci, F. Greco, G. Ianniruberto, Integral and differential constitutive equations for entangled polymers with simple versions of CCR and force balance on entanglements, Rheol. Acta 40 (2) (2001) 98103.

[24] A. Leygue, A.N. Beris, R. Keunings, A constitutive equation for entangled linear polymers inspired by reptation theory and consistent with nonequilibrium thermodynamics, J. Non Newton. Fluid Mech. 101 (2001) $95-111$.

[25] D.W. Mead, R.G. Larson, M. Doi, A molecular theory for fast flows of entangled polymers, Macromolecules 31 (22) (1998) 78957914.

[26] G. Marrucci, G. Ianniruberto, Flow-induced orientation and stretching of entangled polymers, Philos. Trans. R. Soc. A 361 (2003) 677687.

[27] P. Wapperom, R. Keunings, Impact of decoupling approximation between stretch and orientation in rheometrical and complex flow of entangled polymers, J. Non Newton. Fluid Mech. 122 (1-3) (2004) 3343.

[28] F. Léonardi, J.-C. Majesté, A. Allal, G. Marin, Rheological models based on the double reptation mixing rule: the effects of a polydisperse environment, J. Rheol. 44 (2000) 675-692.

[29] C. Pattamaprom, R.G. Larson, T.J. Van Dyke, Quantitative predictions of linear viscoelastic rheological properties of entangled polymers, Rheol. Acta 39 (6) (2000) 517-531.

[30] S.J. Park, R.G. Larson, Tube dilution and reptation in binary blends of monodisperse linear polymers, Macromolecules 37 (2004) 597604

[31] M. Denberg, Study of the stretch dynamics of the CRAFT model using a Monte-Carlo method. Technical Report, Université Catholique de Louvain, 2004. 
[32] A.N. Beris, B.J. Edwards, Thermodynamics of Flowing Systems with Internal Microstructure, Oxford University Press, 1994.

[33] A.N. Beris, Personal communication.

[34] P. Wapperom, R. Keunings, G. Ianniruberto, Prediction of rheometrical and complex flows of entangled linear polymers using the DCR model with chain stretch, J. Rheol. 47 (2003) 247-265.
[35] P.K. Bhattacharjee, J.P. Oberhauser, G.H. McKinley, L.G. Leal, T. Shridar, Extensional rheometry of entangled solutions, Macromolecules 35 (2002) 10131-10148.

[36] A. Bach, K. Almdal, H.K. Rasmussen, O. Hassager, Elongational viscosity of narrow molar mass distribution polystyrene, Macromolecules 36 (14) (2003) 5174-5179. 